\title{
IDENTIFICATION AND CHARACTERIZATION OF A SUBSET OF MOUSE SENSORY NEURONS THAT EXPRESS ACID-SENSING ION CHANNEL 3
}

\author{
Y.-W. LIN, ${ }^{a}$ M.-Y. MIN, ${ }^{b}$ C.-C. LIN, ${ }^{a}$ W.-N. CHEN, ${ }^{a}$ \\ W.-L. WU, ${ }^{a}$ H.-M. YU ${ }^{c}$ AND C.-C. CHEN $^{\mathrm{a} *}$ \\ alnstitute of Biomedical Sciences, Academia Sinica, Taipei 115, Tai- \\ wan, Republic of China \\ ${ }^{b}$ Department of Life Science and Institute of Zoology, College of Life \\ Science, National Taiwan University, Taipei 106, Taiwan, Republic of \\ China \\ ${ }^{\circ}$ Genomic Research Center, Academia Sinica, Taipei 115, Taiwan, \\ Republic of China
}

\begin{abstract}
Acid-sensing ion channel 3 (ASIC3) is the most sensitive acid sensor in sensory neurons that innervate into skin, muscle, heart, and visceral tissues. ASIC3 is involved in ischemia sensing, nociception, mechanosensation, and hearing, but how ASIC3-expressing neurons differ in their firing properties is still unknown. We hypothesized that ASIC3expressing neurons have specialized firing properties, which, coupled with the heterogeneity of acid-sensing properties, accounts for various physiological roles. Here, we successfully identified ASIC3-expressing lumbar dorsal root ganglion (DRG) neurons whose transient proton-gated currents were blocked by salicylic acid (SA). The salicylic acidsensitive (SAS) neurons did not exist in DRG neurons of mice lacking ASIC3. SAS neurons expressed distinct electrophysiological properties as compared with other DRG neurons. Especially, SAS neurons fired action potentials (APs) with large overshoot and long afterhyperpolarization duration, which suggests that they belong to nociceptors. SAS neurons also exhibited multiple nociceptor markers such as capsaicin response $(38 \%)$, action potential (AP) with inflection $(35 \%)$, or tetrodotoxin resistance $(31 \%)$. Only in SAS neurons but not other DRG neurons was afterhyperpolarization duration correlated with resting membrane potential and AP duration. Our studies reveal a unique feature of ASIC3-expressing DRG neurons and a basis for their heterogeneous functions. () 2008 IBRO. Published by Elsevier Ltd. All rights reserved.
\end{abstract}

Key words: ASIC3, DRG, electrophysiology, salicylic acid, pain.

${ }^{*}$ Corresponding author. Tel: +886-2-2652-3917; fax: +886-2-27899224.

E-mail address: chih@ibms.sinica.edu.tw (C.-C. Chen).

Abbreviations: ACSF, artificial cerebrospinal fluid; AHP, afterhyperpolarization; AP, action potential; ASIC3, acid-sensing ion channel 3; $\mathrm{ASIC}^{-1-}$, acid-sensing ion channel 3 knockout; cap + , capsaicinpositive; cap-, capsaicin-negative; DRG, dorsal root ganglion; hASIC3, human acid-sensing ion channel 3; IB4, isolectin B4; NAC, no acid-evoked current; PCR, polymerase chain reaction; SA, salicylic acid; SARS, salicylic acid-resistant with sustained acid-evoked current; SART, salicylic acid-resistant with transient acid-evoked current; SAS, salicylic acid sensitive; TRPV1, capsaicin receptor; TTX, tetrodotoxin; TTX-r, tetrodotoxin resistant; TTX-s, tetrodotoxin sensitive; $\tau$, desensitization time.
Sensing tissue acidosis is an important function for primary sensory afferents in many physiological and pathological states such as metabolism, ischemia and inflammation. Acid-sensing ion channel 3 (ASIC3) is predominantly expressed in sensory neurons and is the most sensitive channel for extracellular $\mathrm{pH}$ decrease (Waldmann et al., 1997). ASIC3 is capable of detecting a narrow range of acidic $\mathrm{pH}$ (7.3-6.7) or lactic acid (in $\mathrm{mM}$ ) in extracellular medium and depolarizing sensory neurons (Immke and McCleskey, 2001). Upon stimulation, ASIC3 mediates a transient or biphasic (transient and sustained) inward current. Thus, ASIC3 is an important molecular sensor for ischemic pain, such as in angina, intermittent claudication, sickle cell anemia, and McArdle's disease (Sutherland et al., 2001; Yagi et al., 2006).

Multiple subunits must assemble to form a functional homomeric ASIC3 channel or a heteromeric channel with other ASIC subtypes (Benson et al., 2002; de la Rosa et al., 2002; Jiang et al., 2006). The ASIC family includes 7 subtypes-ASIC1a, ASIC1b, ASIC2a, ASIC2b, ASIC3, ASIC4, and ASIC5-all expressed in sensory neurons (Waldmann and Lazdunski, 1998; Chen et al., 1998; Akopian et al., 2000; Sakai et al., 1999). Although ASIC3 is the major subtype in cardiac dorsal root ganglion (DRG) afferent neurons, the composition of these ASIC subtypes in most sensory neurons is still unknown (Sutherland et al., 2001).

Identifying and characterizing DRG neurons expressing ASIC3 is of great interest; because of its channel properties and anatomical expression, ASIC3 is involved in many physiological and pathological functions. Immunostaining studies and retrograde labeling revealed ASIC3 localized in several specialized sensory nerve endings of skin, muscle, arteries, inner ear, and visceral tissues (Price et al., 2001; Yiangou et al., 2001; Molliver et al., 2005; Sugiura et al., 2005; Groth et al., 2006; Jiang et al., 2006; Mercado et al., 2006; Hughes et al., 2007). Therefore, ASIC3 has been implicated in nociception, metaboreception, mechanosensation and hearing. Studies of mouse models with ASIC3 dysfunction further support these physiological roles (Price et al., 2001; Chen et al., 2002; Sluka et al., 2003, 2007; Hildebrand et al., 2004; Page et al., 2005; Carter et al., 2005). Although ASIC3 is involved in many different sensory modalities, how ASIC3-expressing sensory neurons behave in their firing properties is unknown. We hypothesized the existence of functionally distinct neuron groups expressing ASIC3.

In vivo studies have revealed that DRG neurons represent more than 25 functionally diverse afferent popula- 
tions specialized in sensory modality, including 12 or more involved in nociception (lggo, 1985; Cooper and Sessel, 1993; Mense, 1993; Schaible and Grubb, 1993; Cervero, 1994). Nociceptive neurons respond to noxious mechanical, heat, and/or cold stimuli, and they can be divided into $A \alpha / \beta, A \delta$, and $C$ nociceptors depending on their range of conducting velocity (Leem et al., 1993; Fang et al., 2005). However, such nociceptive properties are difficult to measure in acute-dissociated DRG neurons. Alternatively, somatic action potential (AP) configuration has been useful in identifying nociceptive neurons in vivo. Longer AP duration and slower maximum rate of fiber firing are the common properties graded according to the conducting velocity $(C>A \delta>A \alpha / \beta)$. In individual velocity groups, nociceptive neurons show a larger AP overshoot and longer afterhyperpolarization (AHP) duration than non-nociceptive lowthreshold mechanoreceptive neurons (Lawson, 2002; Fang et al., 2005). Interestingly, both AP overshoot and AHP duration have similar values in $C, A \delta$, and $A \alpha / \beta$ nociceptive neurons. Therefore, these electrophysiological criteria seem useful for identifying neurons as nociceptive in vitro.

To identify DRG neurons expressing ASIC3, we examined whether the acid-induced inward current could be inhibited by salicylic acid (SA). SA, known for its antiinflammatory and analgesic drug action through inhibition of cyclooxygenase activity, also directly inhibits acidevoked inward currents and APs in many sensory neurons, including nociceptors (Voilley et al., 2001). So far, only ASIC3 homomeric or ASIC3/ASIC2b heteromeric channels are known to be salicylic acid sensitive (SAS). In this study, we compared the AP configuration and acid-evoked currents of SAS neurons and other DRG neurons and demonstrated that ASIC3 was indeed expressed in a subset of lumbar DRG neurons with distinct firing property.

\section{EXPERIMENTAL PROCEDURES}

\section{Molecular cloning and heterologous expression of ASIC3}

Human acid-sensing ion channel 3 (hASIC3) was cloned from human placental cDNA by polymerase chain reaction (PCR). The human cDNA was obtained from placental mRNA by reverse transcription with use of Superscript III Reverse Transcriptase (Invitrogen, Carlsbad, CA, USA). PCR involved a primer $68 \mathrm{bp}$ upstream of the start codon (5'-GGCCTCCTGAATCCTATCTTA$\left.3^{\prime}\right)$ and a primer downstream of the stop codon (5'-TAGGACTTTATTTGGGGTGAA-3') by TaqDNA polymerase with the addition of $1 \mathrm{M}$ Betaine (Invitrogen). After subcloning in pGEM-T EASY vector (Promega, Madison, WI, USA), clones were sequenced on both strands. For expression in mammalian cells, hASIC3 on pGEM-T EASY vector was cut by Notl on both ends and ligated to pIRES-hrGFP-2a vector (Strategene, La Jolla, CA, USA). The recombinant hASIC3 was transfected into COS-7 cells by use of lipofectamine according to the manufacturer's instructions (Invitrogen). Briefly, $5 \mu$ l of lipofectamine in $100 \mu$ l of serum-free DMEM was mixed with $100 \mu \mathrm{l}$ of serum-free medium containing $2 \mu \mathrm{g}$ of hASIC3-pIRES-hrGFP-2a, and then incubated at room temperature for $30 \mathrm{~min}$. The mixture was added to cells, which had been washed twice with serum-free medium. The cells were incubated at $37{ }^{\circ} \mathrm{C}$ in a $\mathrm{CO}_{2}$ incubator for $5 \mathrm{~h}$, and the transfection was stopped with $20 \%$ FCS containing DMEM medium. Whole-cell patch recording was performed the next day.

\section{Animals and preparation of DRG primary cultures}

Adult CD1 mice (8-12 weeks old) were used for these experiments. Acid-sensing ion channel 3 knockout $\left(\mathrm{ASIC}^{-1-}\right.$ ) mice were generated as previously described (Chen et al., 2002) and backcrossed to the CD1 wild type for at least eight generations. All procedures followed the Guide for the Use of Laboratory Animals (National Academy Press, Washington, DC, USA) and were approved by the Institutional Animal Care and Use Committee of Academia Sinica. We did our best to minimize the number of animals used. Mice $(n=30)$ were killed by use of $\mathrm{CO}_{2}$ to minimize their suffering. Lumbar (L1-L5) DRG neurons were dissected bilaterally and placed in a tube containing DMEM and then transferred to DMEM containing type 1 collagenase $(0.125 \%, 90 \mathrm{~min})$ and trypsin $(0.25 \%, 20 \mathrm{~min})$ for digestion at $37^{\circ} \mathrm{C}$. After being washed and trituration, recovered cells were plated on poly-Llysine-coated cover slides. Before whole-cell patch recording, cells were stained with isolectin B4 (IB4) -FITC (4 $\mu \mathrm{g} / \mathrm{ml})$ in solutions containing $\mathrm{MgCl}_{2}, \mathrm{CaCl}_{2}$ and $\mathrm{MnCl}_{2}$ (all in $0.1 \mathrm{mM}$ ) for 2 min. Neurons binding to IB4 were small to medium in size $(<34 \mu \mathrm{m})$ (Fig. 1A). All recordings were completed within $24 \mathrm{~h}$ after plating. A total of $440 \mathrm{DRG}$ neurons were patch recorded.

\section{Whole-cell patch-clamp recording}

Glass pipettes (Warner Products 64-0792) were prepared (1-5 $\mathrm{M} \Omega$ ) with use of a vertical puller (NARISHIGE PP-830). Whole-cell recordings involved use of an Axopatch MultiClamp 700B (Axon Instruments). Stimuli were controlled and digital records captured with use of Signal 3.0 software and a CED1401 converter (Cambridge Electronic Design). Cells with a membrane potential more positive than $-40 \mathrm{mV}$ were not accepted. The bridge was balanced in current clamping recording and series resistance was compensated $70 \%$ in voltage-clamping recording with Axopatch 700B compensation circuitry.

\section{Drugs and solutions}

Recording cells were superfused in artificial cerebrospinal fluid (ACSF) containing (in mM) $130 \mathrm{NaCl}, 5 \mathrm{KCl}, 1 \mathrm{MgCl}_{2}, 2 \mathrm{CaCl}_{2}, 10$ glucose, and 20 Hepes, adjusted to $\mathrm{pH} 7.4$ with $\mathrm{NaOH}$. ACSF solutions were applied by use of gravity. The recording electrodes were filled with (in $\mathrm{mM}$ ) $100 \mathrm{KCl}, 2 \mathrm{Na}_{2}$-ATP, $0.3 \mathrm{Na}_{3}$-GTP, 10 EGTA, $5 \mathrm{MgCl}_{2}$, and 40 Hepes, adjusted to $\mathrm{pH} 7.4$ with $\mathrm{KOH}$. Osmolarity was approximately $300-310 \mathrm{mOsm}$. SA was prepared from a 1-M stock solution (in 100\% ethanol) to a final concentration of $500 \mu \mathrm{M}$ in ACSF. Capsaicin was prepared from a 10-mM stock solution (in $100 \%$ ethanol) to a final concentration of $1 \mu \mathrm{M}$ in ACSF. The final concentration of ethanol was $<0.1 \%$. The $\mathrm{pH} 5.0$ ACSF was titrated by 2-[N-morpholino]ethanesulfonic acid (MES). Tetrodotoxin (TTX) was prepared from a $100-\mu \mathrm{M}$ stock solution to a final concentration of $1 \mu \mathrm{M}$ in ACSF. All drugs were purchased from Sigma Chemical (St. Louis, MO, USA), but TTX was from Tocris (Avonmouth, UK). APETx2 was synthesized by use of a peptide synthesizer (ABI 433A). The synthesized APETx2 concentration was likely to have been overestimated due to decay.

\section{SA inhibition}

The equation used to fit the dose-response curve of $S A$ inhibition was $y=A 2+(A 1-A 2) /\left(1+(x / x 0)^{p}\right)$. A1 means the initial value, $A 2$ means the final value, $x 0$ means the center (point of inflection) and $p$ means the power that affects the slope of the area about the inflection point. We used $100 \%$ as the maximal response to fit the data. 
A

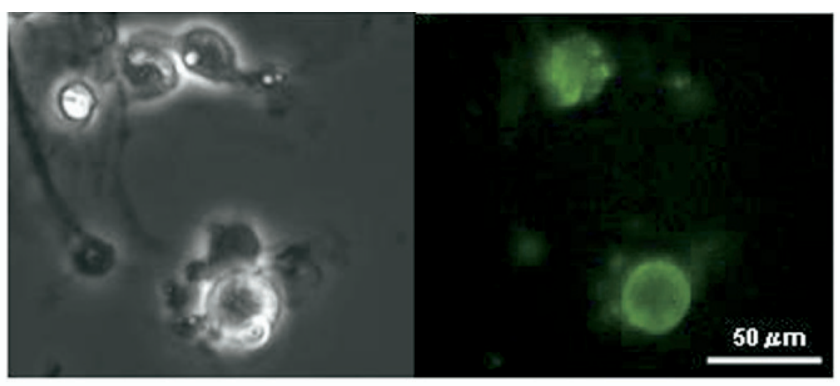

B
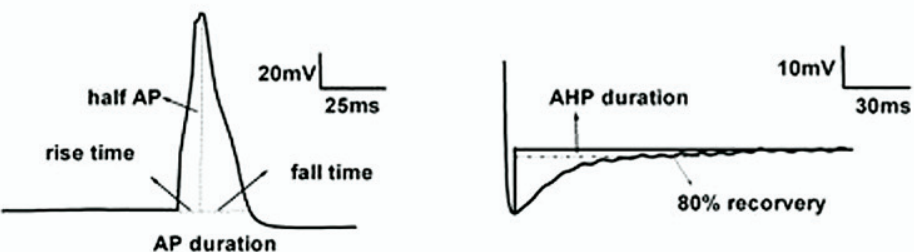

AP duration
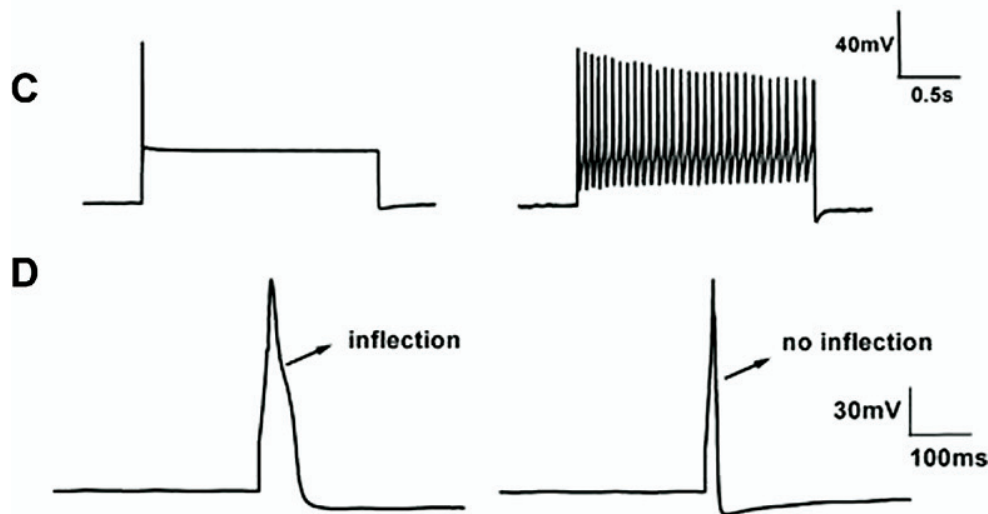

E no-TTX

TTX
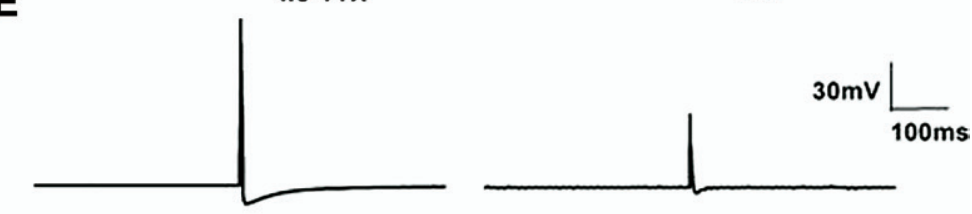

$\mathbf{F}$

no-TTX

TTX
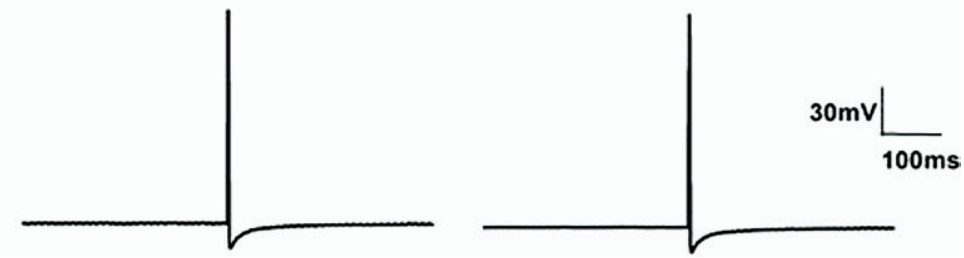

Fig. 1. Nociceptor markers and AP configuration of DRG neurons. (A) Cultured DRG cells in phase contrast (left panel) and fluorescence images after FITC-IB4 labeling (right panel). IB4-positive cells indicated with arrows. (B) Configuration of AP and AHP. (C) Spike patterns of AP; 1-nA square pulse was delivered to define firing patterns with either a single spike or multiple spikes. (D) AP with inflection or no inflection. (E) In TTX-s neurons, a 1-ms 2-nA current injection evoked an AP, which was blocked by TTX. (F) In TTX-r neurons, AP was not affected by TTX treatment.

\section{AP parameters}

First, a 1-ms 2-nA current step was used to determine AP and AHP (Fig. 1B). Then a 50-ms current step was used to determine AP threshold. Then, a 1-nA square pulse was delivered for $2 \mathrm{~s}$ to define spike pattern (Fig. 1C). We determined AP duration, AP rise time, AP fall time, AP overshoot, and AHP duration as described in Fig. 1B. To quantify AHP, we used a criterion of $80 \%$ recovery to baseline (AHP80) (Lawson et al., 1997). Inflections were determined by differentiation of AP (Fig. 1D). All AP parameters were determined under current clamp mode.

\section{ASIC parameters}

After AP parameter detection, we switched to voltage clamp mode to detect acid-induced inward current. The pH 5.0 ACSF solution was 
applied through a glass pipette $50 \mu \mathrm{m}$ from the neuron and via gravity controlled by a VC- 6 six-channel valve controller (Warner Instruments). Acid solution was applied for $4 \mathrm{~s}$ in $30-\mathrm{s}$ intervals. After three applications of acid solution, SA $(500 \mu \mathrm{M})$ was bath applied to examine whether the acid-evoked current was blocked. Then, capsaicin $(1 \mu \mathrm{M})$ responses were tested by the same gravity-controlled system. A neuron was defined as capsaicin positive while capsaicin application induced an inward current larger than 20 pA.

\section{TTX sensitivity}

At the last step of recording, we tested whether the neurons' AP was sensitive to TTX blockade in current clamp mode. Voltagegated $\mathrm{Na}^{+}$channels in the primary sensory neurons are important for generation of AP, and these channels can be distinguished pharmacologically by their relative sensitivity to TTX (Renganathan et al., 2001). In tetrodotoxin-sensitive (TTX-s) neurons, a 1-ms 2-nA current injection evoked a standard AP, which was blocked by TTX (Fig. 1E). In contrast, tetrodotoxin-resistant (TTX-r) neurons fired AP on TTX treatment (Fig. 1F).

\section{Statistical analysis}

Results are presented as means \pm standard errors of means. ASIC rise times were computed as the time between $20 \%$ and $80 \%$ of the peak current observed. The desensitization time $(\tau)$ was de- rived from the expression $\mathrm{A}_{1} \exp \left[-(t \times k) / \tau_{1}\right]+\mathrm{C}$ by use of Origin 5.0 (Microcal Origin Instruments). Points between $10 \%$ of the peak current and $90 \%$ of the return to baseline were fitted by use of Origin 5.0. Linear regression analysis involved the expression $\mathrm{Y}=\mathrm{A}+\mathrm{B} \times \mathrm{X}$. One-way ANOVA test was applied to independent sample comparisons. A $P<0.05$ was considered significant.

\section{RESULTS}

\section{Identification of DRG neurons expressing ASIC3}

To test our hypothesis that ASIC3 is expressed in a functionally distinct group of DRG neurons, we aimed to examine whether this subset of DRG neurons had a unique electrophysiological property. So far, the only specific ASIC3 blocker is the sea anemone peptide APETx2, but the source is too limited to screen ASIC3-positive neurons from a pool of DRG neurons (Diochot et al., 2004, 2007). SA is also a selective blocker for ASIC3 but not for other ASIC subunits (Voilley et al., 2001). We tested whether SA could be a selective blocker for ASIC3-expressing sensory neurons. SA inhibited ASIC3mediated transient proton-gated inward current as well as APETx2 in cells heterologously expressing ASIC3 (Fig. 2A).

A

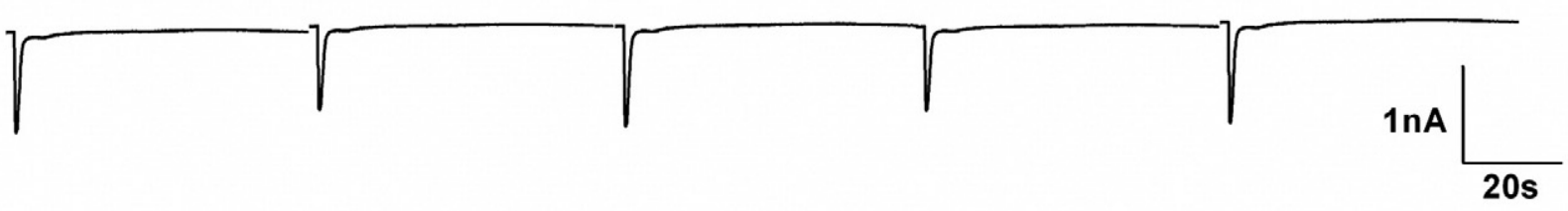

B
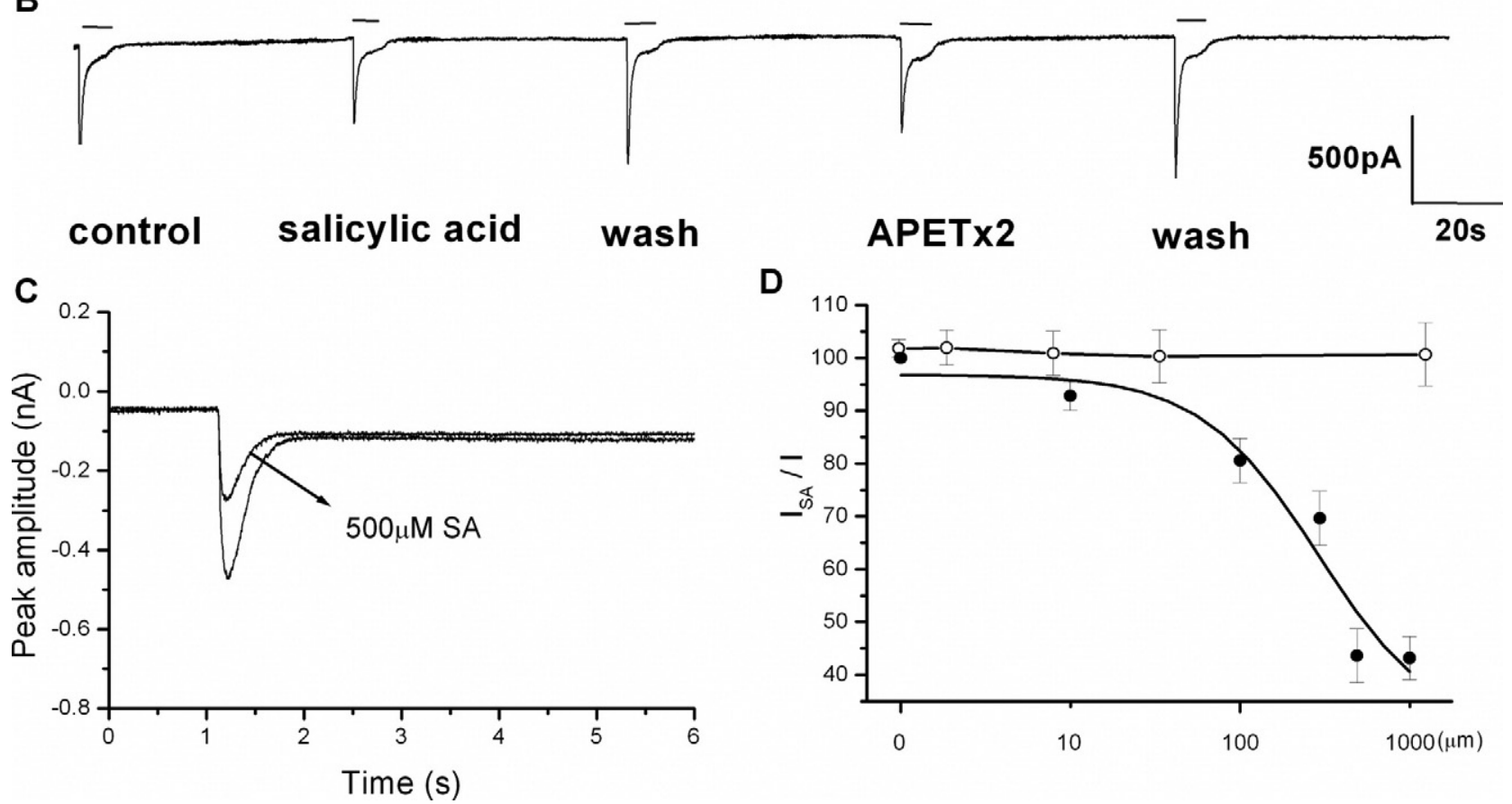

D

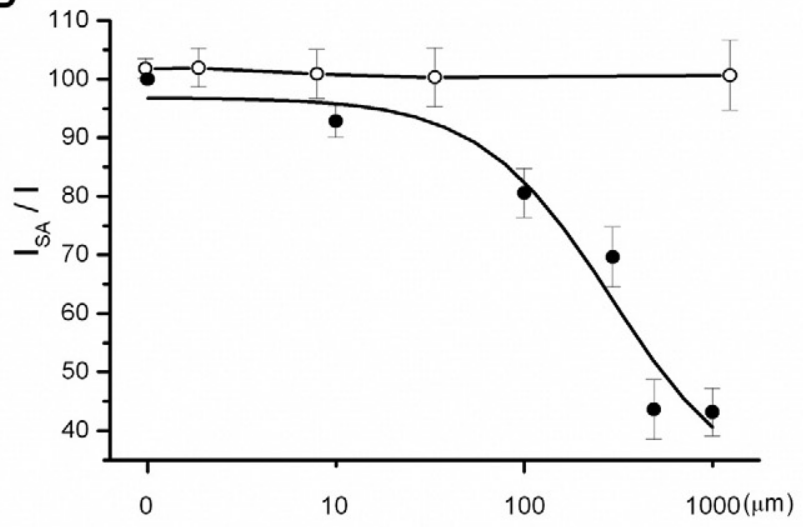

Fig. 2. SA is a selective blocker for ASIC3. (A) SA (500 $\mu \mathrm{M})$ and APETx2 $(1 \mu \mathrm{M})$ inhibited acid-evoked transient inward current in COS-7 cells expressing hASIC3. Acid ( $\mathrm{pH}$ 5.0) was bath applied for $4 \mathrm{~s}$. (B) SA also inhibited acid-evoked inward current in mouse DRG neurons sensitive to APETx2. (C) Representative current traces showed that SA inhibited the transient but not sustained component of proton-gated inward current in SAS neurons. (D) SAS neurons with maximum SA inhibition more than $33 \%$ were chosen for pharmacological studies. Dosage-dependent inhibition of transient proton-gated inward current by SA showed an $\mathrm{IC}_{50}$ of $294 \mu \mathrm{M}$ in SAS neurons, whose maximum SA inhibition occurred at $500 \mu \mathrm{M}$ (filled circles for transient component; open circles for sustained component; $n=13$ ). 
Likewise, SA inhibited acid-induced currents in mouse DRG neurons that were sensitive to APETx2 (Fig. 2B). Thus, we considered that a DRG neuron was an ASIC3expressing sensory neuron when a transient proton-gated inward current was sensitive to SA inhibition. In the SAS neurons, acid ( $\mathrm{pH}$ 5.0) evoked either a transient or biphasic inward current, whereas only the transient inward current was inhibited by SA with $\mathrm{IC}_{50}$ of $294 \mu \mathrm{M}$ (Fig. 2C-D). In 13 tested SAS neurons, $500 \mu \mathrm{M}$ SA achieved maximal inhibition $(56.4 \pm 5.1 \%$ at $500 \mu \mathrm{M}$ SA; $56.8 \pm 4.1 \%$ at $1000 \mu \mathrm{M} \mathrm{SA}$ ), so this concentration was used to determine whether a neuron was SAS. In such neurons, $500 \mu \mathrm{M} \mathrm{SA}$ inhibited a transient proton-gated current ranging from $3 \%$ to $92 \%$. In other DRG neurons, acid evoked a salicylic acid-resistant transient (SART)/biphasic or salicylic acidresistant sustained (SARS) current, or no acid-evoked current (NAC). Accordingly, we classified DRG neurons into four acid-sensitivity groups, depending on their acidevoked current and sensitivity to SA inhibition (Fig. 3A-D).
SA alone at $500 \mu \mathrm{M}$ did not elicit any current in DRG neurons $(n=35)$.

To further verify that SAS neurons represented ASIC3expressing neurons, we examined the existence of SAS neurons in DRG neurons from $\mathrm{ASIC}^{-1-}$ mice. ASIC3 ${ }^{-1-} \mathrm{DRGs}$ contained only SART, SARS, and NAC neurons and no SAS neurons $(n=165)$ (Table 1). The SART neurons had a small transient proton-gated inward current and slow desensitizing kinetics (mean $\tau=1.5 \mathrm{~s}$ ) similar to SART neurons in the wild type (Figs. 3E and 4). In contrast, SAS neurons had a large transient proton-gated inward current and fast desensitizing kinetics (mean $\tau=0.5 \mathrm{~s}$ ) similar to the channel property of ASIC3 (Fig. 4C; Table 2). Therefore, SAS neurons represented a subset of DRG neurons expressing ASIC3.

\section{Distinct electrophysiological properties in SAS neurons}

The SAS neurons showed unique electrophysiological properties as compared with other groups of neurons

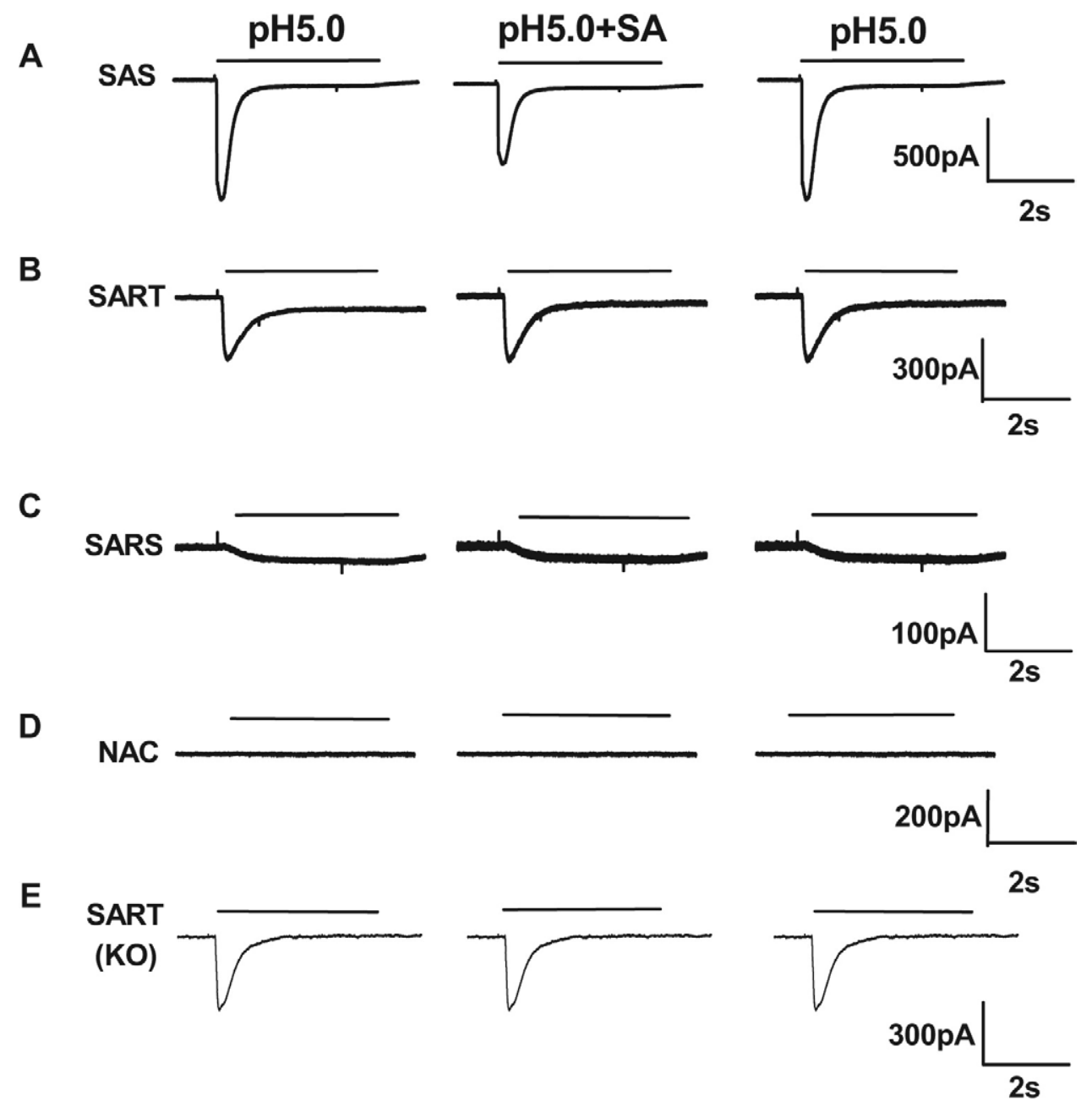

Fig. 3. Four groups of DRG neurons with different sensitivity to acid solution ( $\mathrm{pH}$ 5.0) stimulation and SA inhibition. (A-D) Representative current traces of each acid-evoked current type. Neurons were stimulated by 4-s extracellular acidification of pH 5.0. (A) In SAS neurons, the acid-evoked inward current was inhibited by SA. (B) In SART neurons, acid evoked a transient inward current, which was resistant to SA treatment. (C) In SARS neurons, acid-evoked an SA-resistant sustained inward current. (D) In NAC neurons, NAC was observed. (E) Representative current traces of SART in $\mathrm{ASIC}^{-1-} \mathrm{DRG}$ neurons. The transient proton-gated inward current was resistant to SA inhibition. 
Table 1. Cell numbers of four acid-sensitivity groups in ASIC3 wildtype (WT) and knockout (KO) mice

\begin{tabular}{clcll}
\hline & SAS & SART & SARS & NAC \\
\hline WT $(n=275)$ & & & & \\
$<34 \mu \mathrm{m}$ & $12(4.4 \%)$ & $9(3.3 \%)$ & $57(20.7 \%)$ & $52(18.9 \%)$ \\
$>34 \mu \mathrm{m}$ & $43(15.6 \%)$ & $11(4.0 \%)$ & $53(19.3 \%)$ & $38(13.8 \%)$ \\
KO $(n=165)$ & & & & \\
$<34 \mu \mathrm{m}$ & $0(0 \%)$ & $7(4.2 \%)$ & $38(23.0 \%)$ & $42(25.5 \%)$ \\
$>34 \mu \mathrm{m}$ & $0(0 \%)$ & $23(13.9 \%)$ & $37(22.4 \%)$ & $18(10.9 \%)$ \\
\hline
\end{tabular}

Lumbar-part DRG neurons were classified into four acid-sensitive groups: SAS, SART, SARS and NAC according to acid-evoked current types and SA sensitivity. These neurons were then divided into two cell-size groups (< or $>34 \mu \mathrm{m}$ ). ASIC3 KO showed no SAS neurons. Numbers in parentheses are ratios to total recorded neurons in WT or KO mice.

(Table 2). They had a similar firing property as SART neurons, but their transient proton-gated current was larger and $\tau$ faster. SAS neurons had a more negative $\mathrm{Vm}$, shorter AP duration and fall time, larger AP overshoot, higher AP threshold, and longer AHP duration than both SARS and NAC neurons. As well, SAS neurons had a larger capacitance than SARS neurons. Interestingly, the proportion of neurons responding to capsaicin was $38 \%$ for SAS neurons, the highest percentage in comparison with SART $(10 \%)$, SARS (29\%), and NAC (0\%) neurons. In contrast, SAS neurons had the lowest proportion of multiple spikes $(9.1 \%)$ and TTX resistance $(30.9 \%)$ in the AP configuration than the other three groups of neurons.
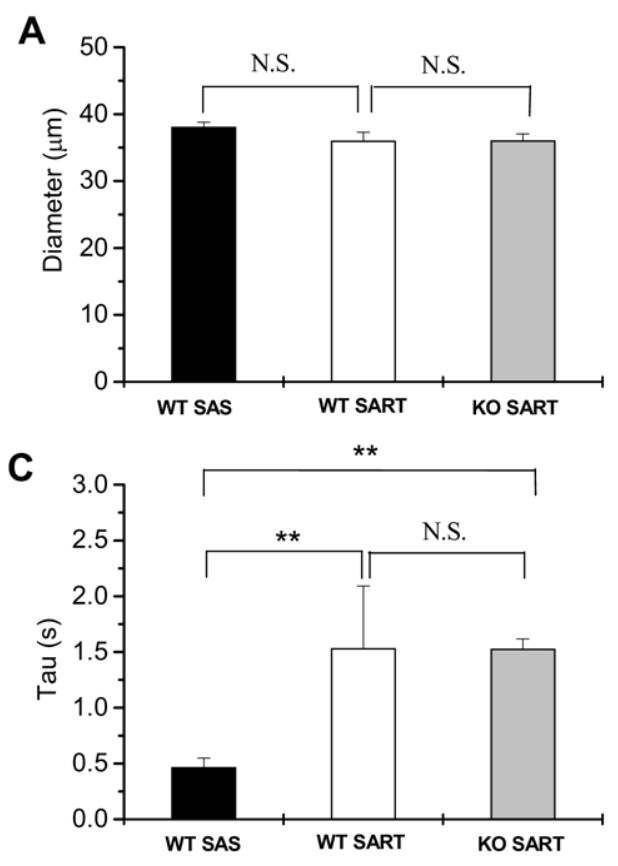

Most SAS neurons did not bind to IB4, a cell marker for small-diameter non-peptidergic nociceptors (Bennett et al., 1998; Stucky and Lewin, 1999). The diameter of SAS neurons ranged from 27 to $50 \mu \mathrm{m}$, with mean size $38.1 \pm 0.7 \mu \mathrm{m}$, which was similar to diameters for the SART group $(35.9 \pm 1.4 \mu \mathrm{m})$ but larger than those for SARS $(34.4 \pm 0.7 \mu \mathrm{m})$ and NAC $(33.1 \pm 0.8 \mu \mathrm{m})$ groups. Because the cell size is usually related to the firing property of DRG neurons either sensitive or resistant to TTX (TTX-s and TTX-r) blockade, we showed the relation between diameter and cell number in TTX-r and TTX-s groups by histography (Fig. 5). As expected, most TTX-r neurons had smaller cell sizes than TTX-s neurons. In both SARS and NAC groups, the TTX-r neurons were largely found in cells with size smaller than $34 \mu \mathrm{m}(31 / 51$ and 27/41, respectively). In contrast, only $31 \%(17 / 55)$ of SAS neurons were TTX-r, and $70 \%(12 / 17)$ of them were in medium-sized cells $(34-42 \mu \mathrm{m})$.

Since most SAS neurons were medium to large size, we wondered whether cell size explained the differences in electrophysiological features. We divided these neurons into small-medium $(<34 \mu \mathrm{m})$ and medium-large $(>34 \mu \mathrm{m})$ groups, because in DRGs from CD1 mice all IB4-positive neurons were smaller than $34 \mu \mathrm{m}$ in diameter. As expected, many AP variables varied by cell size in all four acid-sensitivity groups, especially AP duration and AP threshold (Tables 3-4). Nevertheless, SAS neurons still showed a unique electrophysiological feature as compared with other DRG neurons in either small-medium or medium-
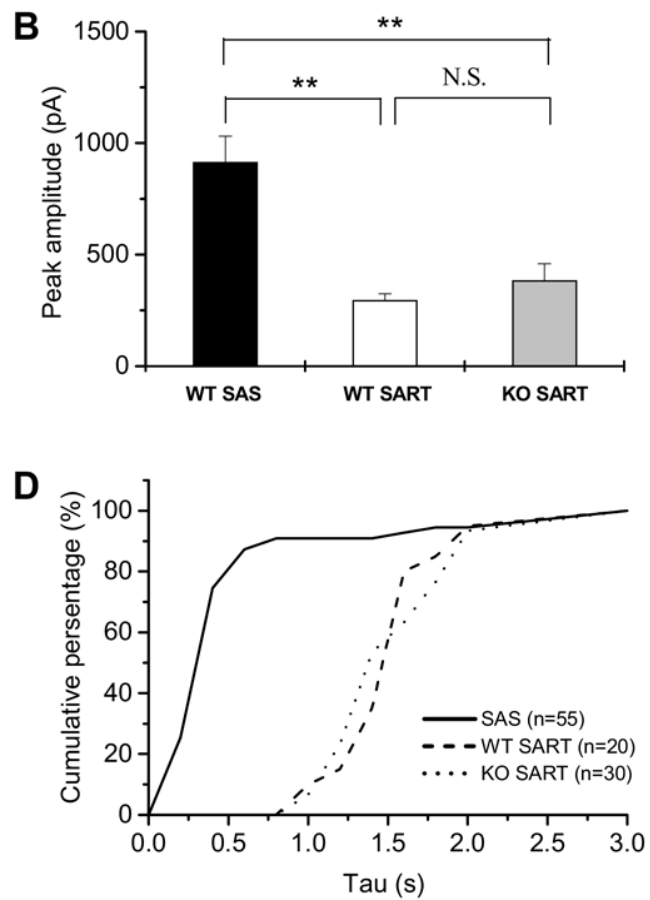

Fig. 4. Neurons with transient proton-gated currents in $A S I C 3^{-1-}$ DRG neurons. (A) Mean cell sizes of SAS neurons and SART neurons. Among $\mathrm{ASIC}^{-1-}$ neurons, no SAS neurons were found. The remaining neurons with transient proton-gated current were SART neurons with cell size similar to that of SAS and SART neurons in wild-type mice. (B) The peak amplitude of transient proton-gated current in SAS neurons was significantly larger than that for both the wild type and $\mathrm{ASIC}^{-1-}$ SART neurons, with no difference in amplitude between the wild-type and ASIC3 ${ }^{-1-}$ SART neurons. (C) The $\tau$ constant of SAS neurons was significantly faster than that for both the wild-type and ASIC3 ${ }^{-1-}$ SART neurons. (D) The $\tau$ constant for the ASIC3 $^{-1-}$ SART neurons was similar to that for the wild type in cumulative percentage. ${ }^{* *} P<0.01$, N.S. not significant. 
Table 2. Electrophysiological properties of four acid-sensitivity groups of lumbar DRG neurons

\begin{tabular}{|c|c|c|c|c|}
\hline & SAS $(n=55)$ & SART $(n=20)$ & SARS $(n=110)$ & NAC $(n=90)$ \\
\hline Diameter $(\mu \mathrm{m})$ & $38.1 \pm 0.7$ & $35.9 \pm 1.4$ & $34.4 \pm 0.7^{* *}$ & $33.1 \pm 0.8^{* *}$ \\
\hline Capacitance (pF) & $31.5 \pm 1.8$ & $25.3 \pm 2.8$ & $26.2 \pm 1.2^{*}$ & $26.8 \pm 1.5$ \\
\hline $\mathrm{Vm}(\mathrm{mV})$ & $-55.5 \pm 1.0$ & $-53.1 \pm 1.8$ & $-50.9 \pm 0.9^{* *}$ & $-47.5 \pm 0.9^{* *}$ \\
\hline $\mathrm{AP}(\mathrm{ms})$ & $6.4 \pm 0.3$ & $8.8 \pm 2.5$ & $7.9 \pm 0.7^{*}$ & $7.8 \pm 0.5^{*}$ \\
\hline Half AP (ms) & $3.1 \pm 0.2$ & $2.7 \pm 0.2$ & $3.3 \pm 0.2$ & $3.7 \pm 0.2$ \\
\hline $\mathrm{AP}$ rise time (ms) & $2.2 \pm 0.1$ & $2.2 \pm 0.2$ & $2.1 \pm 0.1$ & $2.4 \pm 0.1$ \\
\hline AP fall time (ms) & $4.2 \pm 0.3$ & $6.7 \pm 2.6$ & $5.8 \pm 0.7^{*}$ & $5.4 \pm 0.5^{\star}$ \\
\hline Overshoot (mV) & $47.9 \pm 3.7$ & $53.6 \pm 4.4$ & $39.9 \pm 2.0^{*}$ & $35.1 \pm 2.2^{\star *}$ \\
\hline AP threshold $(p A)$ & $578.8 \pm 46.9$ & $455.8 \pm 75.3$ & $395.2 \pm 38.3^{*}$ & $424.0 \pm 38.8^{*}$ \\
\hline $\mathrm{AHP}(\mathrm{ms})$ & $187.1 \pm 17.5$ & $161.4 \pm 24.3$ & $117.7 \pm 10.1^{* *}$ & $102.2 \pm 9.4^{* *}$ \\
\hline Multiple spike (\%) & 9.1 & 15 & 27.3 & 16.7 \\
\hline TTXr (\%) & 30.9 & 40 & 46.4 & 45.6 \\
\hline Inflection (\%) & 34.6 & 20 & 35.5 & 33.3 \\
\hline Capsaicin (\%) & 38.2 & 10 & 29.1 & 0 \\
\hline IB4 (\%) & 5.5 & 5 & 33.6 & 28.9 \\
\hline ASIC rising time (ms) & $62.4 \pm 6.3$ & $68.7 \pm 4.6$ & NA & NA \\
\hline Desensitization time (s) & $0.5 \pm 0.1$ & $1.5 \pm 0.6^{* *}$ & NA & NA \\
\hline Inhibition by SA (\%) & $39.8 \pm 3.3$ & NA & NA & NA \\
\hline Peak amplitude (pA) & $912.8 \pm 117.3$ & $294.1 \pm 30^{* *}$ & $112.3 \pm 15.9^{\star *}$ & NA \\
\hline Current density $(\mathrm{pQ})$ & $867.7 \pm 98.9$ & $636.8 \pm 83.3$ & $345.3 \pm 43.5^{\star *}$ & NA \\
\hline
\end{tabular}

Four acid-sensitivity groups exhibited distinct electrophysiological properties. Vm, resting membrane potential; ASIC, acid-sensing ion channel. Only among SAS neurons was the acid-evoked current sensitive to SA inhibition. The SAS neurons showed unique electrophysiological properties as compared with other groups. NA: nonapplicable.

${ }^{*} P<0.05$ compared with SAS values.

${ }^{* *} P<0.01$ compared with SAS values.

large cell-size groups (Fig. 6). SAS neurons showed a significantly large AP overshoot than NAC neurons in small-medium cells and SARS and NAC neurons in mediumlarge cells (Fig. 6A-D, E, I). In both cell-size groups, SAS neurons showed a significantly longer AHP duration than SARS and NAC neurons (Fig. 6A-D, F, J). Only the SAS $(47.9 \pm 7.9 \mathrm{mV})$ and SARS neurons $(43.8 \pm \mathrm{mV})$ in the small-medium cell-size group showed no significant difference in AP overshoot. SAS and SART neurons shared many similarities in AP configuration in both cell-size groups. SAS neurons showed a larger transient protongated current than SART neurons only in medium-large neurons but faster desensitization kinetics than SART neurons in both cell-size groups (Fig. 6G, H, K, L).

\section{Correlation of AP and ASIC parameters in SAS neurons}

Since AP overshoot, AHP duration, and SA inhibition were characteristic markers for SAS neurons, we next asked whether these three parameters were related to other biophysical variables in DRG neurons. In this study, linear fit was used to compare the relation of AP and ASIC parameters. AP overshoot was positively correlated with AHP duration in SAS, SART, and NAC neurons but not in SARS neurons (Fig. 7A). Only in SAS neurons but not other acid-sensitivity groups was AHP duration positively correlated with $\mathrm{AP}$ duration and negatively correlated with $\mathrm{Vm}$ (Fig. 7B, C). In SAS neurons, the rise time and peak amplitude of transient proton-gated inward current were negatively and positively correlated with AP duration, re- spectively, but correlations were not found in SART neurons (Fig. 7D, E). The rise time of the transient protongated inward current was negatively correlated with peak amplitude (Fig. 7F). Interestingly, SA inhibition was not significantly correlated with any AP parameters. Instead, SA inhibition was negatively correlated with cell diameter and positively correlated with rise time of the transient proton-gated inward current in small-medium SAS neurons (Fig. 8A, B). These correlations were not found in mediumlarge SAS neurons (Fig. 8C, D).

\section{DISCUSSION}

In this study, we demonstrated SA as a reliable selective blocker identifying ASIC3-expressing neurons in mouse DRG neurons. First, SA blocked the APETx2-sensitive transient proton-gated current in DRG neurons and cells heterologously expressing ASIC3. Second, the transient proton-gated current in SAS neurons showed fast desensitizing kinetics similar to that of the ASIC3 channel. Third, ASIC3 $^{-1-}$ mice showed no SAS DRG neurons. Although we cannot exclude the possibility that some ASIC3-expressing neurons have a transient or sustained protongated current resistant to SA inhibition, SAS neurons at least represent a subset of ASIC3-expressing DRG neurons.

We also revealed that ASIC3-expressing SAS neurons were a functionally unique subset (55/275) of lumbar DRG neurons. SAS neurons had different kinetics of acidevoked current and AP configuration as compared with neurons of other acid-sensitivity groups. Furthermore, SAS 
A

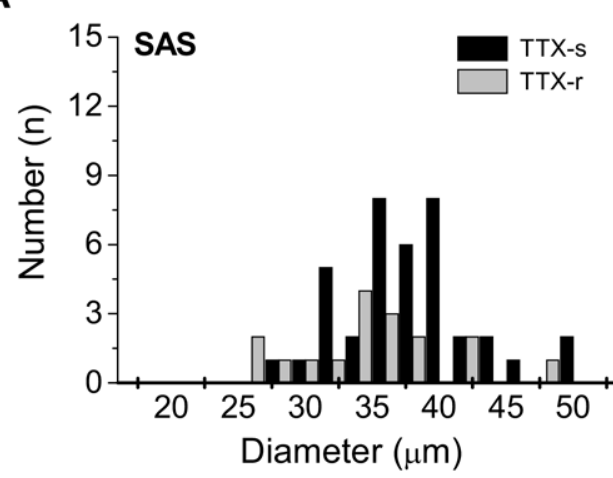

C

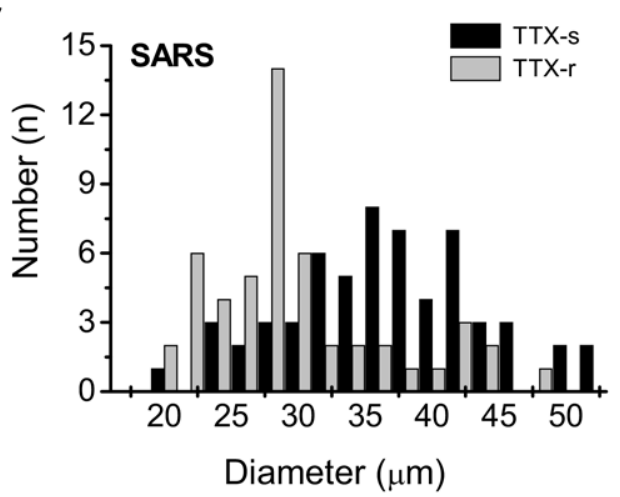

B
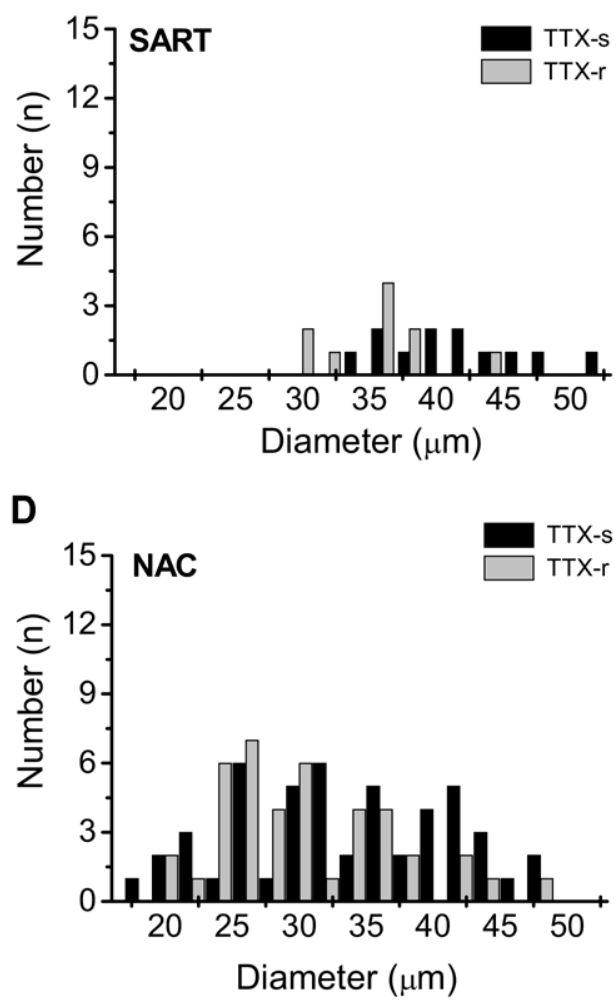

Fig. 5. Histograms of DRG neurons by cell size and TTX sensitivity. (A) SAS neurons. (B) SART neurons. (C) SARS neurons. (D) NAC neurons.

neurons were composed of a heterogeneous population differing in cell size, nociceptor markers, acid-evoked current, SA inhibition, resting membrane potential, and AP configuration.

\section{SA inhibition}

Previous study has demonstrated that SA inhibits both transient and sustained components of ASIC-type currents in DRG neurons (Voilley et al., 2001). However, our results only showed that SA only inhibited the transient protongated current (Fig. 2). We did not know what caused the discrepancy, although it may be due to the different recording systems or using different species of animals (Leffler et al., 2006). The average inhibition with SA (500 $\mu \mathrm{M})$ in SAS neurons was $39.8 \%$, which was less than that of ASIC3/ ASIC2b heteromultimers (66\% inhibition) and ASIC3 homomeric channels ( $>80 \%$ inhibition) in heterologous expression systems (Voilley et al., 2001). Only $5 \%$ of all 80 recorded SAS neurons reached $80 \%$ inhibition by $500 \mu \mathrm{M}$ $\mathrm{SA}$. Therefore, our results favored a predominant expression of heteromeric ASIC3 channels in DRG neurons. Previously, single-channel recording by outside-out patches has demonstrated that most ASIC3 containing channels in DRGs have a channel property similar to that of ASIC2and3 co-expression in oocytes (de la Rosa et al., 2002). The maximum inhibition of SA varied among 13 tested SAS neurons, but they all had a similar $I_{50}$ of SA near $294 \mu \mathrm{M}$. The $\mathrm{IC}_{50}$ of SA for SAS neurons was similar to that in a previous study, which found an $\mathrm{IC}_{50}$ of $260 \mu \mathrm{M}$ in COS-7 cells transfected with ASIC3 (Voilley et al., 2001). The inhibition of SA on ASIC3 seems comparable between DRG neurons and heterologous expression systems. In contrast, APETx2 blocks homomeric ASIC3 in heterologous systems with an $\mathrm{IC}_{50}$ of $63 \mathrm{nM}$, but blocks native channels in DRG neurons with an $\mathrm{IC}_{50}$ of $216 \mathrm{nM}$, which indicates an influence of different membrane environments and/or ASIC composition between cell lines (e.g. COS-7 cells) and neurons (Diochot et al., 2004, 2007). Nevertheless, SA inhibition of ASIC3-like currents seems to depend more on ASIC composition than membrane environment (Voilley et al., 2001). However, the precise ASIC composition in SAS neurons is difficult to obtain because the channel kinetics of different ASIC compositions does not match the native channel properties in DRG neurons, except for the ASIC3 homomeric channels and ASIC2/ASIC3 heteromeric channels (Sutherland et al., 2001; Benson et al., 2002; de la Rosa et al., 2002; Hesselager et al., 2004). This situation may be partially explained by some auxiliary proteins that interact with ASIC3 and other ASIC subunits to alter the native channel properties (Wemmie et al., 2006). Alternatively, the native channels with ASIC-like currents may contain a variety of ASIC compositions. Classification of DRG neurons by the current signature method followed by immunostaining supports that ASIC3-containing neurons express heterogeneous ASIC subunits (Jiang et al., 2006). Moreover, certain clusters of ASIC subunits or auxiliary proteins may 
Table 3. Electrophysiological properties of four acid-sensitivity groups of lumbar DRG neurons $(>34 \mu \mathrm{m})$

\begin{tabular}{|c|c|c|c|c|}
\hline & SAS $(n=43)$ & SART $(n=10)$ & SARS $(n=53)$ & NAC $(n=38)$ \\
\hline Diameter $(\mu \mathrm{m})$ & $40.0 \pm 0.6$ & $40.0 \pm 1.5$ & $41.2 \pm 0.7$ & $40.6 \pm 0.7$ \\
\hline Capacitance (pF) & $32.8 \pm 2.1$ & $27.0 \pm 4.7$ & $32.2 \pm 1.8$ & $36.9 \pm 2.4$ \\
\hline $\mathrm{Vm}(\mathrm{mV})$ & $-56.5 \pm 1.0$ & $-55.8 \pm 2.0$ & $-54.5 \pm 1.1$ & $-51.8 \pm 1.4^{\star *}$ \\
\hline $\mathrm{AP}(\mathrm{ms})$ & $6.0 \pm 0.3$ & $6.3 \pm 0.9$ & $4.8 \pm 0.3^{*}$ & $6.0 \pm 0.5$ \\
\hline Half AP (ms) & $3.0 \pm 0.2$ & $2.7 \pm 0.3$ & $2.5 \pm 0.2$ & $3.1 \pm 0.2$ \\
\hline $\mathrm{AP}$ rise time (ms) & $2.3 \pm 0.1$ & $2.3 \pm 0.3$ & $2.0 \pm 0.1$ & $2.3 \pm 0.1$ \\
\hline AP fall time (ms) & $3.8 \pm 0.3$ & $3.9 \pm 0.8$ & $2.7 \pm 0.3^{\star *}$ & $3.7 \pm 0.5$ \\
\hline Overshoot (mV) & $47.9 \pm 4.3$ & $51.1 \pm 7.6$ & $35.7 \pm 3.1^{*}$ & $38.7 \pm 3.9^{*}$ \\
\hline AP threshold (pA) & $600.2 \pm 53.7$ & $623.1 \pm 103.3$ & $598.3 \pm 57.5$ & $618.7 \pm 65.2$ \\
\hline $\mathrm{AHP}(\mathrm{ms})$ & $183.0 \pm 19.0$ & $153.4 \pm 38.8$ & $110.9 \pm 15.3^{* *}$ & $120.4 \pm 6.7^{\star *}$ \\
\hline Multiple spike (\%) & 4.7 & 0 & 3.7 & 7.9 \\
\hline $\operatorname{TTXr}(\%)$ & 27.9 & 18.1 & 22.6 & 36.8 \\
\hline Inflection (\%) & 27.9 & 20.0 & 11.3 & 18.4 \\
\hline Capsaicin (\%) & 39.5 & 18.8 & 26.4 & 0 \\
\hline IB4 (\%) & 0 & 0 & 0 & 0 \\
\hline ASIC rising time (ms) & $55.5 \pm 3.7$ & $68.7 \pm 8.1$ & NA & NA \\
\hline Desensitization time (s) & $0.4 \pm 0.1$ & $1.4 \pm 0.1^{* *}$ & NA & NA \\
\hline Inhibition by SA (\%) & $40.8 \pm 3.6$ & NA & NA & NA \\
\hline Peak amplitude (pA) & $977.6 \pm 131.5$ & $296.7 \pm 49.7^{\star *}$ & $112.3 \pm 15.9^{\star *}$ & NA \\
\hline Current density (pQ) & $884.1 \pm 95.6$ & $583.7 \pm 95.6$ & $345.3 \pm 43.5^{\star \star}$ & NA \\
\hline
\end{tabular}

Four acid-sensitivity groups exhibited distinct electrophysiological properties (cell size larger than $34 \mu \mathrm{m}$ in diameter). SA, salicylic acid; SAS, salicylic acid sensitive; SART, salicylic acid resistant with transient acid-evoked current; SARS, salicylic acid resistant with sustained acid-evoked current; NAC, no acid-evoked current. Only among SAS neurons was the acid-evoked current sensitive to SA inhibition. The SAS neurons showed unique electrophysiological properties as compared with other groups. NA: nonapplicable.

${ }^{*} P<0.05$ compared with SAS values.

${ }^{* *} P<0.01$ compared with SAS values.

exist in small-medium SAS neurons because they showed significant correlations between SA inhibition and cell diameter or rise time of transient proton-gated current (Fig. 8).
Future studies with single-cell RT-PCR might provide a clue to the molecular heterogeneity of the SAS neurons (Liss and Roeper, 2004).

Table 4. Electrophysiological properties of four acid-sensitivity groups of lumbar DRG neurons $(<34 \mu \mathrm{m})$

\begin{tabular}{|c|c|c|c|c|}
\hline & SAS $(n=12)$ & SART $(n=10)$ & SARS $(n=57)$ & NAC $(n=52)$ \\
\hline Diameter $(\mu \mathrm{m})$ & $31.1 \pm 0.7$ & $31.0 \pm 1.0$ & $28.1 \pm 0.5^{*}$ & $27.7 \pm 0.5^{\star}$ \\
\hline Capacitance (pF) & $26.7 \pm 2.7$ & $23.3 \pm 2.6$ & $20.6 \pm 1.0^{*}$ & $19.5 \pm 1.0^{*}$ \\
\hline $\mathrm{Vm}(\mathrm{mV})$ & $-51.9 \pm 3.0$ & $-49.9 \pm 2.9$ & $-47.7 \pm 1.3$ & $-44.4 \pm 1.1^{* *}$ \\
\hline $\mathrm{AP}(\mathrm{ms})$ & $7.8 \pm 0.8$ & $11.9 \pm 5.5$ & $10.9 \pm 1.0$ & $9.2 \pm 0.8$ \\
\hline Half AP (ms) & $3.8 \pm 0.6$ & $2.8 \pm 0.4$ & $4.1 \pm 0.2$ & $4.2 \pm 0.3$ \\
\hline AP rise time (ms) & $2.0 \pm 0.1$ & $1.9 \pm 0.2$ & $2.2 \pm 0.1$ & $2.4 \pm 0.1$ \\
\hline AP fall time (ms) & $8.8 \pm 0.7$ & $10.2 \pm 5.6$ & $8.7 \pm 1.0$ & $6.8 \pm 0.7$ \\
\hline Overshoot (mV) & $47.9 \pm 8.0$ & $56.7 \pm 3.2$ & $43.8 \pm 2.4$ & $32.4 \pm 2.6^{* *}$ \\
\hline AP threshold ( $p A)$ & $360.0 \pm 77.6$ & $251.3 \pm 63.9$ & $206.4 \pm 36.7^{\star}$ & $281.8 \pm 36.8$ \\
\hline $\mathrm{AHP}$ (ms) & $200.6 \pm 43.7$ & $171.0 \pm 28.1$ & $123.9 \pm 13.5^{\star *}$ & $88.9 \pm 1.7^{* *}$ \\
\hline Multiple spike (\%) & 25 & 33.3 & 49.1 & 7.9 \\
\hline TTXr (\%) & 41.7 & 66.7 & 68.4 & 36.8 \\
\hline Inflection (\%) & 50 & 10 & 57.9 & 18.4 \\
\hline Capsaicin (\%) & 33.3 & 0 & 26.3 & 0 \\
\hline IB4 (\%) & 25 & 10 & 64.9 & 0 \\
\hline ASIC rising time (ms) & $63.3 \pm 8.7$ & $67.5 \pm 3.9$ & NA & NA \\
\hline Desensitization time (s) & $0.6 \pm 0.3$ & $1.5 \pm 0.2^{* *}$ & NA & NA \\
\hline Inhibition by SA (\%) & $40.8 \pm 3.6$ & NA & NA & NA \\
\hline Peak amplitude (pA) & $633.3 \pm 256.2$ & $352.9 \pm 50.0$ & $101.1 \pm 21.2^{* *}$ & NA \\
\hline Current density (pQ) & $749.8 \pm 307.9$ & $705.7 \pm 144.5$ & $279.1 \pm 46.8^{* *}$ & NA \\
\hline
\end{tabular}

Four acid-sensitivity groups exhibited distinct electrophysiological properties (cell size smaller than $34 \mu \mathrm{m}$ in diameter).Only among SAS neurons was the acid-evoked current sensitive to SA inhibition. The SAS neurons showed unique electrophysiological properties as compared with other groups. NA: nonapplicable.

${ }^{*} P<0.05$ compared with SAS values.

${ }^{* *} P<0.01$ compared with SAS values. 
A

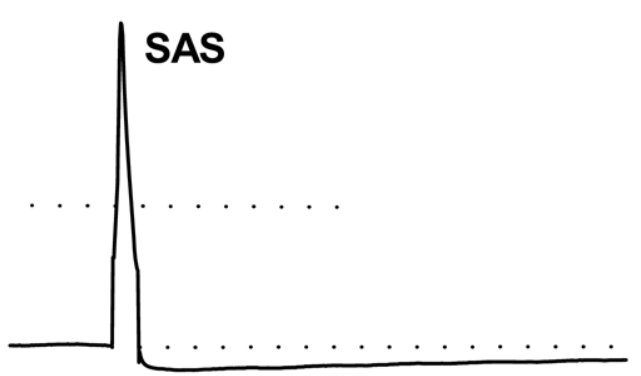

C SARS

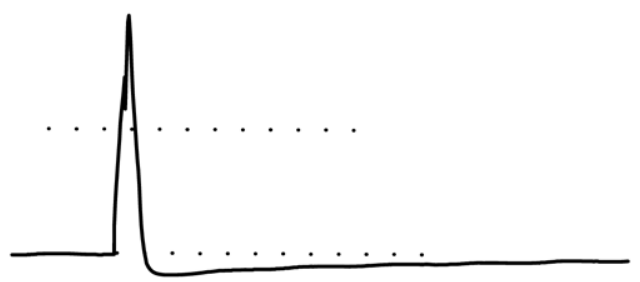

B

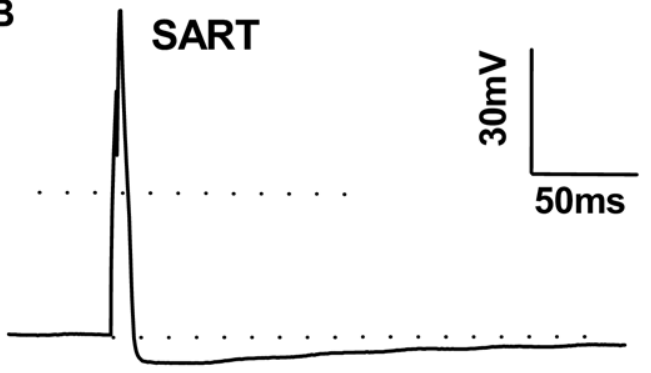

D NAC

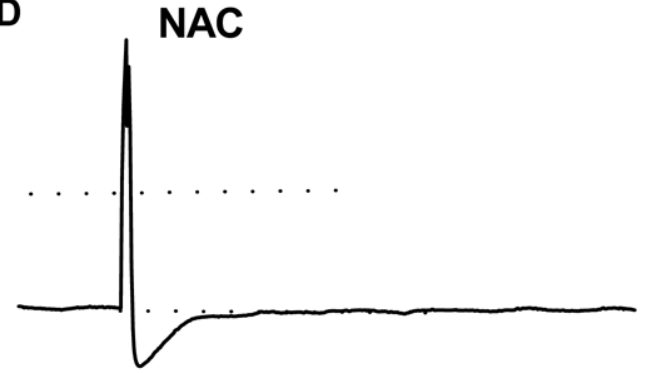

E

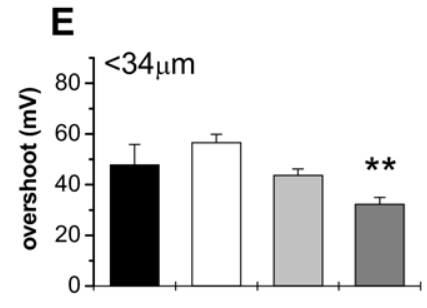

F
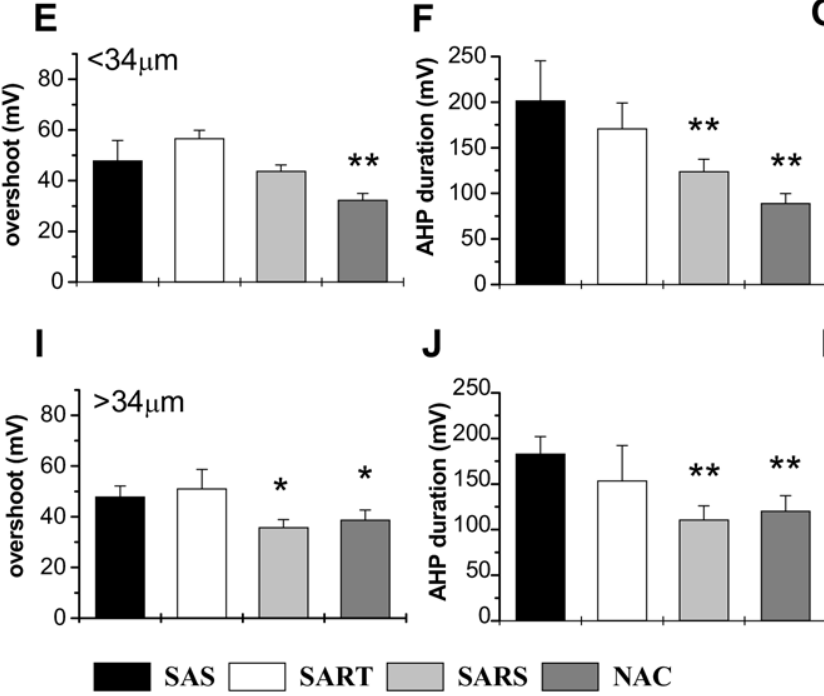

J

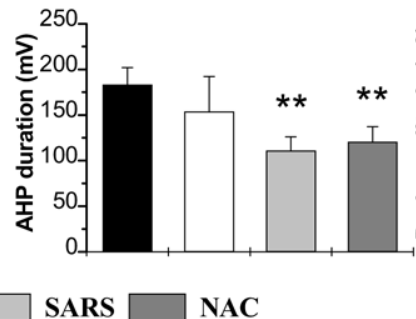

G

H

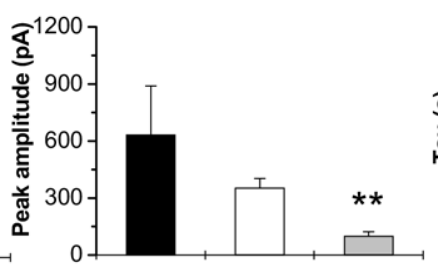

K

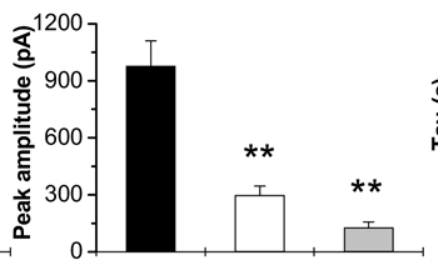

L

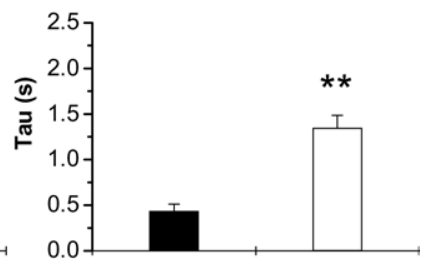

Fig. 6. Electrophysiological properties of DRG neurons with small-medium and medium-large diameter in four acid-sensitivity groups. Representative APs in SAS (A), SART (B), SARS (C), and NAC (D) neurons showed a difference in AP overshoot and AHP duration. Two dotted lines indicate the range between $0 \mathrm{mV}$ and resting membrane potential. $(\mathrm{E}-\mathrm{H})$ Results showing AP overshoot (E), AHP duration (F), peak amplitude $(\mathrm{G})$ and $\tau$ constant (H) of transient proton-gated current of DRG neurons of small-medium diameter in four acid-sensitivity groups. (I-L) Results showing AP overshoot (I), AHP duration $(\mathrm{J})$, peak amplitude $(\mathrm{K})$ and $\tau$ constant $(\mathrm{L})$ of transient-proton-gated current of DRG neurons of medium-large diameter in four acid-sensitivity groups.

\section{Contribution of ASIC3 in transient proton-gated current}

Acid solution (or protons) evoked either transient and/or sustained inward currents in $67.3 \%(185 / 275)$ of neurons in lumbar DRG neurons. The transient proton-gated current was solely mediated by ASIC channels; a sustained proton-gated current can be mediated by TRPV1, TASK, or G-protein-coupled receptors (Reeh and Kress, 2001). The ASIC-mediated currents were found in $27 \%$ $(75 / 275)$ of total DRG neurons and in $16 \%(21 / 130)$ of neurons of small-medium size $(<34 \mu \mathrm{m})$. The relative low expression of ASIC-like currents in DRG neurons of small-medium size in our study (CD1 mice) was also found in studies of C57/BL6 - and Balb/C - mice but not in rats (Leffler et al., 2006). The biophysics of ASIC-like currents was highly heterogeneous in terms of peak amplitude, rise time, and $\tau$ constant. Previous studies have found at least seven ASIC-like current types in sensory neurons (Krishtal and Pidoplichko, 1981; Poirot et al., 2006). We were the first to demonstrate that most of them were ASIC3-containing neurons sensitive to SA inhibition (55/75) in mice. ASIC3 ${ }^{-1-}$ mice did not exhibit SAS DRG neurons, but the total ratio of acid-sensitive DRG neurons was only slightly reduced in $\mathrm{ASIC} 3^{-1-}$ mice 
A

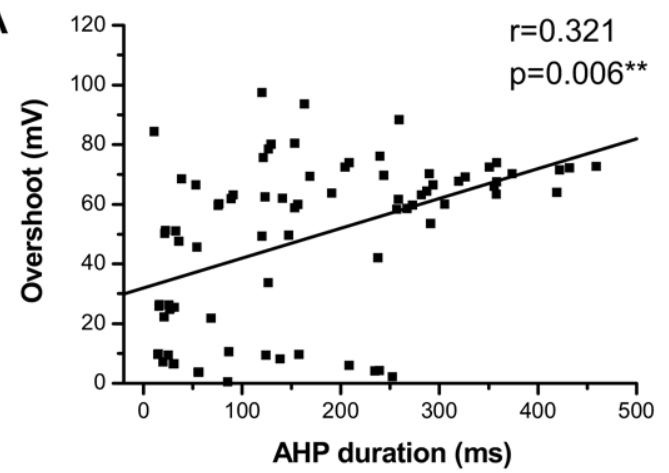

C

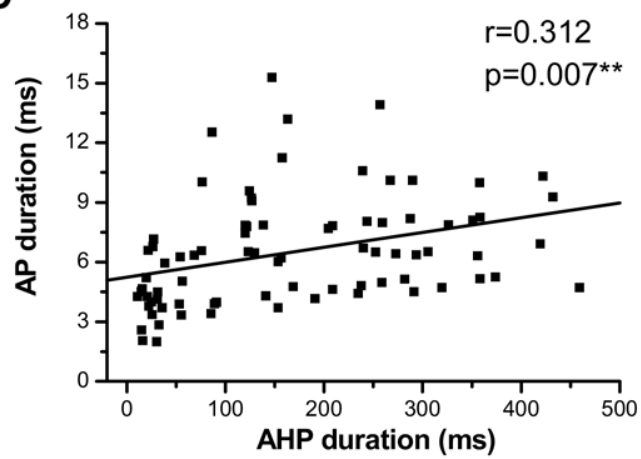

E

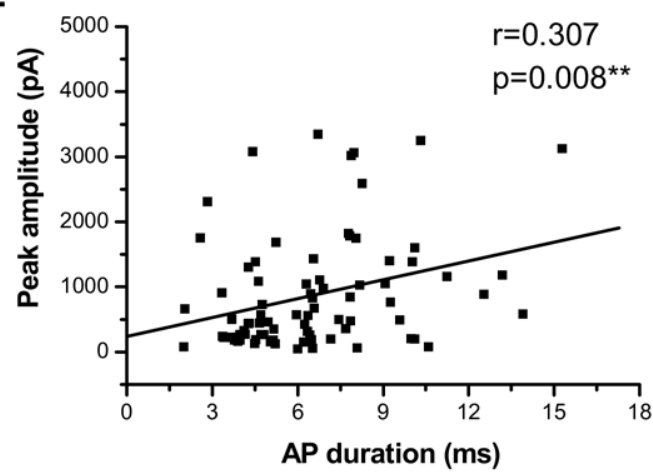

B

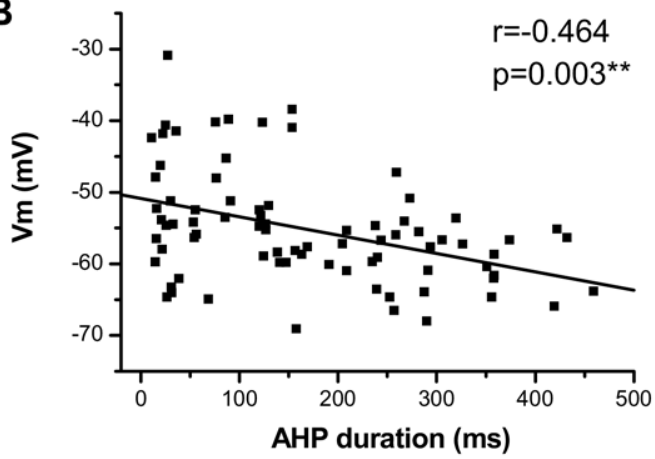

D

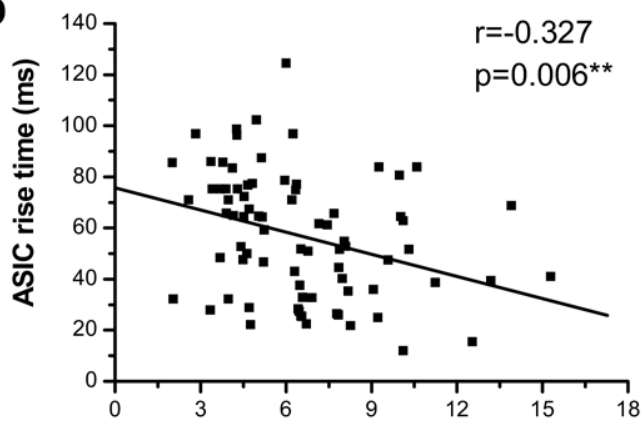

F $\quad 5000 \quad$ AP duration (ms)

$r=-0.364$

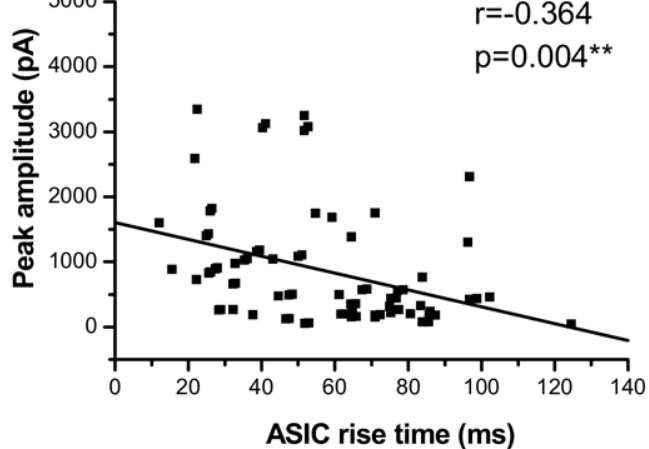

Fig. 7. Linear correlations of AP and ASIC variables in SAS neurons. AHP duration was positively correlated with AP overshoot (A) and AP duration (B), and negatively correlated with $\mathrm{Vm}(\mathrm{C})$. AP duration was negatively correlated with rise time (D) and positively correlated with peak amplitude (E) of the transient proton-gated inward current respectively. The rise time and peak amplitude of the transient proton-gated inward current were negatively correlated (F). ${ }^{* *} P<0.01$.

(63.6\%, 105/165). In ASIC3 ${ }^{-1-}$ mice, the proportion of DRG neurons in SART, SARS, and NAC neurons was all increased (Table 1). Thus, ASIC3 was expressed alone or co-localized with other proton-sensing ion channels/receptors in DRG neurons.

Previous studies have demonstrated that heteromultimeric ASIC subunits form a functional channel to mediate the transient proton-gated currents in DRG neurons (Benson et al., 2002; Xie et al., 2002). The transient protongated current has very fast desensitization kinetics, which is contributed by ASIC3 (Sutherland et al., 2001). In $\mathrm{ASIC}^{-1-}$ mice, the transient proton-gated inward current has a slow desensitizing time constant in DRG neurons (Benson et al., 2002; Xie et al., 2002). In this study, we revealed that ASIC3 might contribute to the transient proton-gated current only in a subset of DRG neurons, SAS neurons. The transient proton-gated current in SART neurons had a slower $\tau$ constant than that in SAS neurons. $\mathrm{ASIC}^{-1-}$ mice exhibited no SAS neurons, and all transient proton-gated currents showed desensitization kinetics like that of SART neurons. However, we did not observe an increase in acid-evoked peak amplitude, as was observed previously (Xie et al., 2002). Instead, the amplitude of the transient proton-gated currents was significantly more decreased in ASIC3 ${ }^{-1-}$ SART neurons than in SAS neurons (Fig. 4A). The number of SART neurons was greatly increased in $A S I C 3^{-1-}$ DRG neurons, but the increased number was far less than the loss of SAS neurons. Therefore, some SAS neurons might express a homomeric ASIC3 channel, so they could contribute to the increased number of SARS and NAC neurons in $\mathrm{ASIC}^{-1-}$ mice. 
A

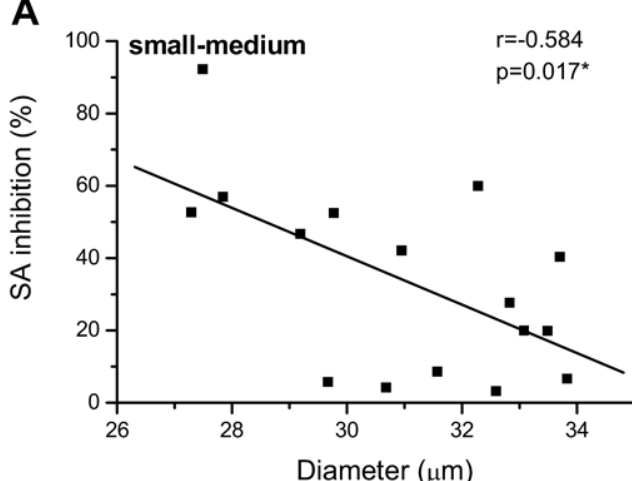

C

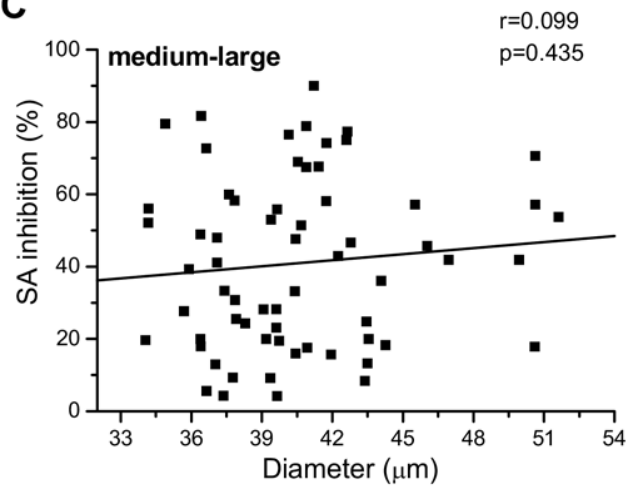

B

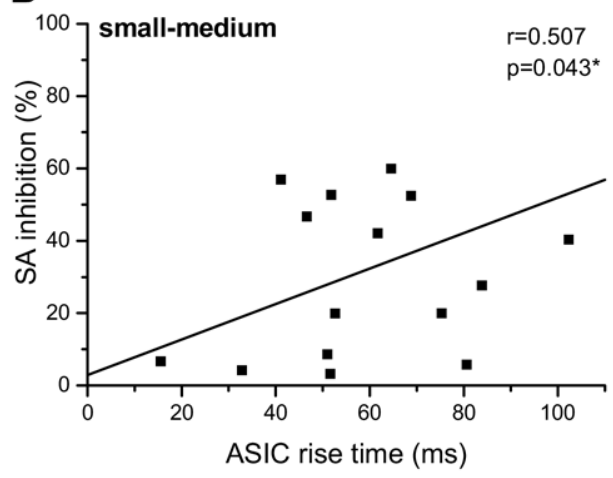

D

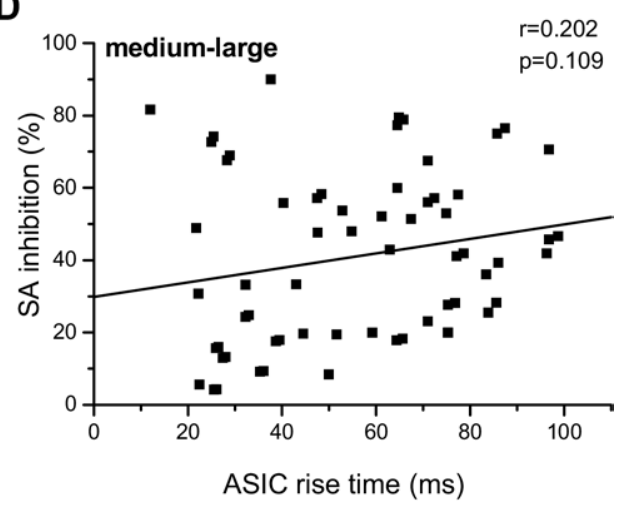

Fig. 8. Linear correlations of SA inhibition with cell diameter and ASIC variables in small-medium and medium-large SAS neurons. (A, B) SA inhibition was negatively correlated with cell diameter $(A)$ and positively correlated with rise time of the transient proton-gated inward current $(B)$ in small-medium SAS neurons. (C, D) SA inhibition in medium-large SAS neurons did not show significant correlation with cell diameter (C) and rise time of the transient proton-gated inward current (D).

\section{Co-localization of ASIC3 and capsaicin receptor (TRPV1) in SAS neurons}

Many capsaicin-sensitive small DRG neurons $(15-30 \mu \mathrm{m})$ generate ASIC-like transient proton-gated currents in response to acid ( $\mathrm{pH} 5.0)$ stimulation in rats $(62 \%)$, but only a few have ASIC-like currents in mice (6\%) (Leffler et al., 2006). In our study of mice, we also found a low portion (8\%) of capsaicin-sensitive small neurons with ASIC-like currents. In contrast, $55 \%$ of capsaicin-sensitive DRG neurons of medium-large size $(>30 \mu \mathrm{m})$ had ASIC-like currents, $90 \%$ of which were SAS neurons. The different contribution of TRPV1 and ASIC subunits to proton sensitivity of sensory neurons accounts for the discrepancy in findings between mice and rats. However, the small size range $(<30 \mu \mathrm{m})$ for nociceptors in rat DRG neurons may not fully reflect the $\mathrm{C}$ - and $\mathrm{A} \delta$-fiber nociceptive populations in mouse DRG neurons, especially in CD1 mice. Nevertheless, capsaicin response was highly associated with ASIC3 expression, and this co-localization is also supported by in situ hybridization results (Ugawa et al., 2005). However, response to capsaicin was not a good marker to sub-classify SAS neurons, because the capsaicin-positive $($ cap +$)$ SAS neurons $(n=21)$ showed similar electrophysiological properties as capsaicin-negative (cap-) SAS neurons $(n=34)$, except for the $\tau$ constant for the ASIC-like current $(0.73 \pm 0.22 \mathrm{~s}$ in cap + vs. $0.31 \pm 0.03 \mathrm{~s}$ in cap - , $P<0.05$, data not shown).

\section{Nociceptive features: AHP and AP overshoot}

In DRG neurons, nociceptive neurons are largely found in slow-conducting neurons of small-medium diameter, but some fast-conducting medium-large neurons are nociceptive as well (Fang et al., 2005). Although most of our SAS neurons were medium-large in diameter (mean size $38.1 \mu \mathrm{m})$ and did not bind to the non-peptidergic nociceptor marker IB4, their AP configuration suggested that they might be pro-nociceptive. Nociceptive neurons usually have a longer AHP duration and larger AP overshoot than low-threshold mechanoreceptive neurons, and these phenomena are not dependent on conducting velocity in vivo (Lawson, 2002; Fang et al., 2005). In our studies, SAS neurons had a significantly longer AHP duration and larger AP overshoot than SARS and NAC neurons. Our results agree with those from previous work showing that DRG neurons with transient proton-gated currents tend to have long AHP duration (Petruska et al., 2002). The long AHP duration of SAS neurons implied that they might correspond to the mechanically insensitive (silent) nociceptors found in in vivo studies (Djouhri et al., 1998). In parallel to this, a previous study of isolated DRG neurons found that ASIC3 does not contribute to a mechanically activated current (Drew et al., 2004), despite discrepant results from ex vivo skin or visceral preparations (Price et al., 2001; Carter et al., 2005). According to the long AHP and large mean cell sizes, SAS neurons may be composed of many 
A $\delta$ - or $A \alpha / \beta$-nociceptive neurons but only a few C-nociceptive neurons.

\section{Heterogeneous SAS neurons}

Previous studies have shown that the ASIC3-positive population in DRG neurons is heterogeneous in terms of cell size, expression of neurotrophic factor receptors and neuropeptides, co-localization with $\mathrm{P} 2 \mathrm{X} 3$ or TRPV1, and tissue innervation (Molliver et al., 2005). In our study, we expanded on findings of this heterogeneity to the firing properties of the SAS neurons. SAS neurons varied in many AP parameters, such as AP duration, AP rise and fall time, AHP duration, and TTX sensitivity, as well as amplitude of transient-proton-gated current. This heterogeneity can be best delineated by classifying SAS neurons by AP inflection, which is mediated by voltage-gated $\mathrm{Ca}^{2+}$ channels and TTX-r Na ${ }^{+}$channels (Blair and Bean, 2002). SAS neurons with AP inflection tended to have longer AP duration, AP fall time, and AHP duration and larger transient proton-gated current than neurons without AP inflection (data not shown). Correlation analyses of AP and ASIC variables further supported that SAS neurons were highly heterogeneous (Figs. 7-8). Besides the heterogeneity of firing properties, voltage-dependent ion channels may exist as clusters in each SAS neuron, since the significant correlations between AHP duration and $\mathrm{Vm}$ or AP duration were unique to SAS neurons but not to other acid-sensitivity groups. The expression profiles of voltage-dependent channels and ASIC subtypes might be subtly controlled in each SAS neuron, as their AP duration was positively correlated with the peak amplitude of transient protongated current.

\section{Clinical implication}

$\mathrm{SA}$ is effective to block low $\mathrm{pH}$-induced cutaneous pain in humans (Steen et al., 1996). However, high doses or chronic exposure to SA causes tinnitus and sepsis-like disease (Guitton et al., 2003; English et al., 1996; Clark et al., 2001). The underlying mechanism of the salicylate toxicity/poisoning is not well understood. Because SA selectively blocks ASIC3 but not other ASIC subtypes, ASIC3-expressing DRG neurons may be the neuronal target for salicylate poisoning in high doses. Therefore, our finding will be of significance clinically, because for the first time we are able to dissect the functional variety of ASIC3expressing DRG neurons and provide a new basis for drug development against ischemic pain and a new concept in treatment of salicylate poisoning.

Acknowledgments-This research was supported by National Science Council grants, NSC 94-2320-B-001-039 and NSC 95-2320B-001-045. Y.-W.L. was supported by a postdoctoral fellowship of Academia Sinica.

\section{REFERENCES}

Akopian AN, Chen CC, Ding YN, Cesare P, Wood JN (2000) A new member of the acid-sensing ion channel family. Neuroreport 11: $2217-2222$.
Bennett DL, Michael DJ, Ramachandran N, Muson JB, Averill S, Yan Q, McMahon SB, Priestley JV (1998) A distinct subgroup of small DRG cells express GDNF receptor components and GDNF is protective for these neurons after nerve injury. J Neurosci 18: 3059-3072.

Benson CJ, Xie J, Wemmie JA, Price MP, Henss JM, Welsh MJ (2002) Heteromultimers of DEG/ENaC subunits form $\mathrm{H}+$-gated channels in mouse sensory neurons. Proc Natl Acad Sci U S A 99: 2338-2343.

Blair NT, Bean BP (2002) Roles of tetrodotoxin (TTX)-sensitive $\mathrm{Na}^{+}$ current, TTX-resistant $\mathrm{Na}^{+}$current, and $\mathrm{Ca}^{2+}$ current in the action potentials of nociceptive sensory neurons. J Neurosci 22:1027710290.

Carter R, Jones W III, Xu L, Gebhart GF (2005) The mechanosensitivity of mouse colon afferent fibers and their sensitization by inflammatory mediators require transient receptor potential vanilloid and acid-sensing ion channel 3. J Neurosci 25:1098110989.

Cervero F (1994) Sensory innervation of the viscera: peripheral basis of visceral pain. Physiol Rev 74:95-137.

Chen CC, England S, Akopian AN, Wood JN (1998) A sensory neuronspecific, proton-gated ion channel. Proc Natl Acad Sci U S A 95:10240-10245.

Chen CC, Zimmer A, Sun WH, Hall J, Brownstein MJ, Zimmer A (2002) A role for ASIC3 in the modulation of high-intensity pain stimuli. Proc Natl Acad Sci U S A 99:8992-8997.

Clark I, Whitten R, Molyneux M, Taylor T (2001) Salicylate, nitric oxide, malaria, and Reye's syndrome. Lancet 357:625-627.

Cooper BY, Sessel BJ (1993) Physiology of nociception in the trigeminal system. In: The headache (Olson J, Tfelt-Hansen P, Welch KMA, eds), pp 87-92. New York: Raven.

de la Rosa DA, Zhang P, Shao D, White F, Canessa CM (2002) Functional implications of the localization and acitivity of acidsensitive channels in rat peripheral nervous system. Proc Natl Acad Sci U S A 99:2326-2331.

Diochot S, Baron A, Rash LD, Deval E, Escoubas P, Scarzello S, Salinas M, Lazdunski M (2004) A new sea anemone peptide APETx2 inhibits ASIC3, a major acid-sensitive channel in sensory neurons. EMBO J 23:1516-1525.

Diochot S, Salinas M, Baron A, Escoubas P, Lazdunski M (2007) Peptides inhibitors of acid-sensing ion channels. Toxicon 49: 271-284.

Djouhri L, Bleazard L, Lawson SN (1998) Association of somatic action potential shape with sensory receptive properties in guinea pig dorsal root ganglion neurones. J Physiol 513:857-872.

Drew LJ, Rohrer DK, Price MP, Blaver KE, Cockayne DA, Cesare P, Wood JN (2004) Acid-sensing ion channels ASIC2 and ASIC3 do not contribute to mechanically activated currents in mammalian sensory neurones. J Physiol 556:691-710.

English M, Marsh V, Amukoye E, Lowe B, Murphy S, Marsh K (1996) Chronic salicylate poisoning and severe malaria. Lancet 347 : $1736-1737$.

Fang X, McMullan S, Lawson SN, Djouhri L (2005) Electrophysiological difference between nociceptive and non-nociceptive dorsal root ganglion neurones in the rat in vivo. J Physiol 565:927-943.

Groth M, Helbig T, Grau V, Kummer W, Haberberger RV (2006) Spinal afferent neurones projecting to the rat lung and pleura express acid-sensitive channels. Respir Res 7:96.

Guitton MJ, Caston J, Ruel J, Johnson RM, Pujol R, Puel JL (2003) Salicylate induces tinnitus through activation of cochlear NMDA receptors. J Neurosci 23:3944-3952.

Hesselager M, Timmermann DB, Ahring PK (2004) pH dependency and desensitization kinetics of heterologously expressed combinations of acid-sensing ion channel subunits. J Biol Chem 279: 11006-11015.

Hildebrand MS, de Silva MG, Klockars T, Rose E, Price M, Smith RJ, McGuirt WT, Christopoulos H, Petit C, Dahl HH (2004) Characterization of DRASIC in the mouse inner ear. Hear Res 190:149-160. 
Hughes PA, Brierley SM, Young RL, Blackshaw LA (2007) Localization and comparative analysis of acid-sensing ion channel (ASIC1, 2 , and 3) mRNA expression in mouse colonic sensory neurons within thoracolumbar dorsal root ganglia. J Comp Neurol 500: $863-875$.

Iggo A (1985) Sensory receptors in the skin of mammals and their sensory functions. Rev Neurol 141:599-613.

Immke DC, McCleskey EW (2001) Lactate enhances the acid-sensing $\mathrm{Na}^{+}$channel on ischemia-sensing neurons. Nat Neurosci 4: 869-870.

Jiang N, Rau KK, Johnson RD, Cooper BY (2006) Proton sensitivity $\mathrm{Ca}^{2+}$ permeability and molecular basis of acid-sensing in channels expressed in glabrous and hairy skin afferents. J Neurophysiol 95:2466-2478.

Krishtal OA, Pidoplichko VI (1981) A receptor for protons in the membrane of sensory neurons may participate in nociception. Neuroscience 6:2599-2601.

Lawson SN, Crepps BA, Perl ER (1997) Relationship of substance P to afferent characteristics of dorsal root ganglion neurones in guineapig. J Physiol 505:177-191.

Lawson SN (2002) Nociceptors as homeostatic afferents: central processing. Exp Physiol 87:239-244.

Leem WJ, Willis WD, Weller SC, Chung JM (1993) Differential activation and classification of cutaneous afferents in the rat. J Neurophysiol 70:2411-2424.

Leffler A, Monter B, Koltzenburg M (2006) The role of the capsaicin receptor TRPV1 and acid-sensing ion channels (ASICs) in proton sensitivity of subpopulations of primary nociceptive neurones in rats and mice. Neuroscience 139:699-709.

Liss B, Roeper J (2004) Correlation function and gene expression of individual basal ganglia neurons. Trends Neurosci 27:475-481.

Mense S (1993) Nociception from skeletal muscle in relation to clinical muscle pain. Pain 54:241-289.

Mercado F, Lopez I, Acuna D, Vega R, Soto E (2006) Acid-sensing ionic channels in the rat vestibular endorgans and ganglia. J Neurophysiol 96:1615-1624.

Molliver DC, Immke DC, Fierro L, Pare M, Rice FL, McCleskey EW (2005) ASIC3, an acid-sensing ion channel, is expressed in metaboreceptive sensory neurons. Mol Pain 1:35.

Page AJ, Brierley SM, Martin CM, Price MP, Symonds E, Butler R, Wemmie JA, Blackshaw LA (2005) Different contributions of ASIC channels $1 \mathrm{a}, 2$, and 3 in gastrointestinal mechanosensory function. Gut 54:1408-1415.

Petruska JC, Napaporn J, Johnson RD, Cooper BY (2002) Chemical responsiveness and histochemical phenotype pf electrophysiologically classified cells of the adult rat dorsal root ganglion. Neuroscience 115:15-30.

Poirot O, Berta T, Decosterd I, Kellenberger S (2006) Distinct ASIC currents are expressed in rat putative nociceptors and are modulated by nerve injury. J Physiol 576:215-234.

Price MP, Mcllwrath SL, Xie J, Cheng C, Qiao J, Tarr DE, Sluka KA, Brennan TJ, Lewin GR, Welsh MJ (2001) The DRASIC cation channel contributes to the detection of cutaneous touch and acid stimuli in mice. Neuron 32:1071-1083.

Reeh PW, Kress M (2001) Molecular physiology of proton transduction in nociceptors. Curr Opin Pharmacol 1:45-51.
Renganathan M, Cummins T, Waxman SG (2001) Contribution of Nav1.8 sodium channels to action potential electrogenesis in DRG neurons. J Neurophysiol 86:629-640.

Sakai H, Lingueglia E, Champigny G, Mattei MG, Lazdunski M (1999) Cloning and functional expression of a novel degenerin-like $\mathrm{Na}^{+}$ channel gene in mammals. J Physiol 519:323-333.

Schaible HG, Grubb BD (1993) Afferent and spinal mechanisms of joint pain. Pain 55:5-54.

Sluka KA, Price MP, Breese NM, Stucky CL, Wemmie JA, Welsh MJ (2003) Chronic hyperalgesia induced by repeated acid injections in muscle is abolished by the loss of ASIC3, but not ASIC1. Pain 106:229-239.

Sluka KA, Radhakrishnan R, Benson CJ, Eshcol JO, Price MP, Babinski K, Audette KM, Yeomans DC, Wilson SP (2007) ASIC3 in muscle mediates mechanical, but not heat, hyperalgesia associated with muscle inflammation. Pain 129:102-112.

Steen KH, Reeh PW, Kreysel HW (1996) Dose-dependent competitive block by topical acetylsalicylic and salicylic acid of low $\mathrm{pH}$-induced cutaneous pain. Pain 64:71-82.

Stucky CL, Lewin GR (1999) Isolectin B4-positive and -negative nociceptors are functionally distinct. J Neurosci 19:6497-6505.

Sugiura T, Dang K, Lamb K, Bielefeldt K, Gebhart GF (2005) Acidsensing properties in rat gastric sensory neurones from normal and ulcerated stomach. J Neurosci 25:2617-2627.

Sutherland SP, Benson CJ, Adelman JP, McCleskey EW (2001) Acidsensing ion channel 3 matches the acid-gated current in cardiac ischemia-sensing neurons. Proc Natl Acad Sci U S A 98:711-716.

Ugawa S, Ueda T, Yamamura H, Shimada S (2005) In situ hybridization evidence for the coexistence of ASIC and TRPV1 within rat sensory neurones. Mol Brain Res 136:125-133.

Voilley N, de Weille J, Mamet J, Lazdunski M (2001) Nonsteroid anti-inflammatory drugs inhibit both the activity and the inflammation-induced expression of acid-sensing ion channels in nociceptors. J Neurosci 21:8026-8033.

Waldmann R, Bassilana F, de Weille J, Champigny G, Heurteaux C, Sakai H, Lazdunski M (1997) Molecular cloning of a non-inactivating proton-gated $\mathrm{Na}^{+}$channel specific for sensory neurons. J Biol Chem 272:20975-20978.

Waldmann R, Lazdunski M (1998) $\mathrm{H}^{+}$-gated cation channels: neuronal acid sensors in the ENaC/DEG family of ion channels. Curr Opin Neurobiol 8:18-424.

Wemmie JA, Price MP, Welsh MJ (2006) Acid-sensing ion channels: advances, questions, and therapeutic opportunities. Trends Neurosci 29:578-585.

Xie J, Price MP, Berger AL, Welsh MJ (2002) DRASIC contributes to $\mathrm{pH}$-gated currents in large dorsal root ganglion sensory neurons by forming heteromultimeric channels. J Neurophysiol 87:2835-2843.

Yagi J, Wenk HN, Naves LA, McCleskey EW (2006) Sustained currents through ASIC3 ion channels at the modest $\mathrm{pH}$ changes that occur during myocardial ischemia. Circ Res 99:501-509.

Yiangou Y, Facer P, Smith JA, Sangameswaran L, Eglen R, Birch R, Knowles C, Williams N, Anand P (2001) Increased acid-sensing ion channel ASIC3 in inflames human intestine. Eur J Gastroenterol Hepatol 13:891-896. 